\title{
Homogenization of boundary value problems for monotone operators in perforated domains with rapidly oscillating boundary conditions of Fourier type
}

\author{
A. Piatnitski ${ }^{b}$, V. Rybalko ${ }^{\sharp}$ \\ October 30, 2018 \\ $b \sharp$ Narvik University College \\ Postboks 385, 8505 Narvik, Norway \\ and \\ P.N.Lebedev Physical Institute of RAS, \\ Leninski pr., 53, Moscow 117924, Russia \\ andrey@sci.lebedev.ru \\ Department of Mathematics, \\ B. Verkin Institute for Low Temperature Physics and Engineering (FTINT) \\ 47 Lenin Ave., Kharkov 61103, Ukraine \\ vrybalko@ilt.kharkov.ua
}

\begin{abstract}
The paper deals with homogenization problem for nonlinear elliptic and parabolic equations in a periodically perforated domain, a nonlinear Fourier boundary conditions being imposed on the perforation border. Under the assumptions that the studied differential equation satisfies monotonicity and 2-growth conditions and that the coefficient of the boundary operator is centered at each level set of unknown function, we show that the problem under consideration admits homogenization and derive the effective model.
\end{abstract}




\section{Introduction}

This paper addresses the homogenization of the boundary value problem

$$
\left\{\begin{array}{l}
-\operatorname{div} a\left(D u_{\varepsilon}, x / \varepsilon\right)+\lambda u_{\varepsilon}=f \text { in } \Omega_{\varepsilon} \\
a\left(D u_{\varepsilon}, x / \varepsilon\right) \cdot \nu=0 \text { on } \partial \Omega \\
a\left(D u_{\varepsilon}, x / \varepsilon\right) \cdot \nu=g\left(u_{\varepsilon}, x / \varepsilon\right) \text { on } S_{\varepsilon}
\end{array}\right.
$$

where $\Omega_{\varepsilon}$ is a bounded periodically perforated domain in $\mathbb{R}^{N}(N \geq 2), \varepsilon>0$ is a small parameter referred to the perforation period. The boundary of $\Omega_{\varepsilon}$ consists of two parts, namely, the fixed outer boundary $\partial \Omega$, and the boundary of perforations $S_{\varepsilon}$. We assume that the domain is not perforated in a small (of order $\varepsilon$ ) neighbourhood of $\partial \Omega$ so that the perforation boundary $S_{\varepsilon}$ and $\partial \Omega$ are disjoint. The coefficients $a=\left(a_{1}, \ldots, a_{N}\right)$ in the equation and the function $g$ in the boundary condition on $S_{\varepsilon}$ are strongly oscillating (with the period $\varepsilon$ ) functions. The boundary condition on $S_{\varepsilon}$ includes, as a particular case, the inhomogeneous Neumann boundary condition of the form $a\left(D u_{\varepsilon}, x / \varepsilon\right) \cdot \nu=\alpha(x / \varepsilon)$ and the Fourier one, $a\left(D u_{\varepsilon}, x / \varepsilon\right) \cdot \nu=\beta\left(u_{\varepsilon}, x / \varepsilon\right) u_{\varepsilon}$. Along with the stationary problem (1.1) we also consider the parabolic problem

$$
\left\{\begin{array}{l}
\partial_{t} u_{\varepsilon}-\operatorname{div} a\left(D u_{\varepsilon}, x / \varepsilon\right)=f \text { in } \Omega_{\varepsilon} \times\{t>0\} \\
a\left(D u_{\varepsilon}, x / \varepsilon\right) \cdot \nu=0 \text { on } \partial \Omega \\
a\left(D u_{\varepsilon}, x / \varepsilon\right) \cdot \nu=g\left(u_{\varepsilon}, x / \varepsilon\right) \text { on } S_{\varepsilon} \\
u_{\varepsilon}=\tilde{u} \text { for } t=0 .
\end{array}\right.
$$

The linear elliptic equations in perforated domains with the Fourier boundary condition on the boundary of perforations were considered, e.g., in [7], [8], [3], 4], [15], [16], 18. It was shown that if the coefficient in the Fourier boundary condition is small (of order $\varepsilon$ ), or the volume fraction of the holes vanishes at a certain rate, as $\varepsilon \rightarrow 0$, then the asymptotic behaviour of solutions to these equations is described in terms of a homogenized problem with an additional potential. By contrast, if the volume fraction of the holes does not vanish as the period of the structure tends to zero, then the dissipative Fourier boundary condition forces solutions vanish.

In the problem studied in the present work the surface measure $\left|S_{\varepsilon}\right|$ tends to infinity as $\varepsilon \rightarrow 0$. To compensate this measure grows we assume that the average 
of the function $g\left(u, x / \varepsilon\right.$ ) (appearing in the boundary condition on $S_{\varepsilon}$ ) over the boundary of each hole is zero for any $u \in \mathbb{R}$.

Previously, linear problems with the same assumptions on the coefficient in the Fourier boundary condition were considered in [5]; related spectral problems were studied in [19],[20]. The corresponding homogenized operator is shown to contain an additional potential, this potential is always negative.

A variational problem closely related to (1.1) for a functional with a bulk energy and a surface term on the perforation boundary was studied in [6] by means of $\Gamma$-convergence technique.

In contrast to [6] we do not assume that the problem under consideration can be written in variational form. Instead, we assume the monotonicity of $a(\xi, y)$ and apply here the celebrated two-scale convergence method (see, e.g. [14], [1], [13]). This allows us to treat boundary value problems that can not be reduced to the minimization of an energy functional; for instance, such a reduction is not possible in the case of linear function $a(\xi, y), a(\xi, y)=A(y) \xi$, with nonsymmetric matrix $A$.

Since, in general, the monotonicity assumption on $a(\xi, y)$ does not imply the monotonicity of the problem (1.1) (even for large $\lambda$ ) we are not able to show the uniqueness result for (1.1). Moreover, the existence of a solution of (1.1) holds only for sufficiently large $\lambda$ (see the discussion in [6]), while the parabolic problem (1.2) does have a unique solution under certain assumptions on $a(\xi, y)$ and $g(u, y)$.

The key difficulty in applying the two-scale convergence theory to the homogenization of (1.1) and (1.2) is due to the presence of a highly perturbed surface integral in the weak formulations of the said problems. To pass to the limit in the surface integral we establish a new result related to the two-scale convergence of traces, see Proposition 7 .

The main result of this work shows that solutions $u_{\varepsilon}$ of problem (1.1) converge as $\varepsilon \rightarrow 0$ to a solution $U_{0}$ of the homogenized problem

$$
\left\{\begin{array}{l}
\operatorname{div} a^{*}\left(D U_{0}, U_{0}\right)+b^{*}\left(D U_{0}, U_{0}\right)+\left|Y^{*}\right|\left(f-\lambda U_{0}\right)=0 \text { in } \Omega \\
a^{*}\left(D U_{0}, U_{0}\right) \cdot \nu=g^{*}\left(U_{0}\right) \cdot \nu \text { on } \partial \Omega .
\end{array}\right.
$$

The coefficients $a^{*}, b^{*}$ are defined in terms of a cell problem (see problem (2.13)) 
and depend both on the coefficients $a=\left(a_{1}, \ldots, a_{N}\right)$ in the equation in (1.1) and on the function $g$ in the boundary condition on $S_{\varepsilon}$. It is interesting to observe also that the homogenization of (1.1) leads to the change of the boundary condition on $\partial \Omega$ from the homogeneous Neumann condition to a Fourier type one.

In what concerns the parabolic problem (1.2), we show that solutions $u_{\varepsilon}$ of (1.2) converge as $\varepsilon \rightarrow 0$ to a solution $U_{0}$ of the homogenized problem

$$
\left\{\begin{array}{l}
\left|Y^{*}\right| \partial_{t} U_{0}-\operatorname{div} a^{*}\left(D U_{0}, U_{0}\right)-b^{*}\left(D U_{0}, U_{0}\right)=\left|Y^{*}\right| f \text { in } \Omega \times\{t>0\} \\
a^{*}\left(D U_{0}, U_{0}\right) \cdot \nu=g^{*}\left(U_{0}\right) \cdot \nu \text { on } \partial \Omega \\
U_{0}=\tilde{u} \text { when } t=0
\end{array}\right.
$$

The analysis of (1.2) involves the same ideas as that of (1.1) combined with a lower semicontinuity trick already used in the parabolic problems in [9, [10, [11, [17.

An interesting issue in both parabolic and elliptic frameworks is the uniqueness of a solution of the limit problem. The limit operator, although admits a priory estimates, need not be monotone even for large values of $\lambda$. The main difficulty is due to the fact that the first order term $b^{*}(D u, u)$ in the limit equation couples the unknown function $u$ and its gradient.

The uniqueness is proved only for small space dimensions and in the case when either $a(\xi, y)$ is linear in $\xi$ or $g(u, y)$ is linear in $u$. Without these additional assumptions it remains an open problem.

The paper is organized as follows. Section 2 is devored to problem setup and formulation of the main results.

Sections 3 15 deal with the elliptic case. In Section 3 we prove the two-scale convergence result which relies on several technical statements. These technical statements are then justified in Sections 4 and 5 ,

Section 6] considers the parabolic case.

Finally, in Section [7 we study the properties of the homogenized problems. 


\section{Presentation of main results}

Let $Y$ be the unit cube $Y=[-1 / 2,1 / 2)^{N}(N \geq 2)$, and let $G$ be an open subset of $Y$ such that $\bar{G} \subset(-1 / 2,1 / 2)^{N}$, with Lipschitz boundary. Set $Y^{*}=Y \backslash G$ and $S=\bigcup_{m \in \mathbb{Z}}(\partial G+m)$.

Given a bounded connected open set $\Omega \subset \mathbb{R}^{N}$ with Lipschitz boundary $\partial \Omega$, we consider the perforated domain $\Omega_{\varepsilon}$ defined by

$$
\Omega_{\varepsilon}=\Omega \backslash \bigcup_{m \in I_{\varepsilon}}(\varepsilon G+m \varepsilon), I_{\varepsilon}=\left\{m \in \mathbb{Z}^{N} ; Y_{\varepsilon}^{(m)} \subset \Omega\right\},
$$

where $Y_{\varepsilon}^{(m)}=(Y+m) \varepsilon$. We have $\partial \Omega_{\varepsilon}=\partial \Omega \cup S_{\varepsilon}$, where $S_{\varepsilon}$ is the boundary of perforations.

We assume that $a: \mathbb{R}^{N} \times Y \rightarrow \mathbb{R}^{N}$ and $g: \mathbb{R} \times S \rightarrow \mathbb{R}$ satisfy

(i) $a(\xi, y)$ (resp. $g(u, y))$ is continuous in $\xi$ (resp. $u)$, i.e. $a \in C\left(\mathbb{R}^{N} ; L^{\infty}(Y)\right)$, $g \in C\left(\mathbb{R} ; L^{\infty}(S)\right)$, and $Y$-periodic in $y$;

(ii) there is $\kappa>0$ such that

$$
(a(\xi, y)-a(\zeta, y)) \cdot(\xi-\zeta) \geq \kappa|\xi-\zeta|^{2} \forall \xi, \zeta \in \mathbb{R}^{N}
$$

(iii) there are constants $C_{1}, \ldots, C_{8}>0$ such that

$$
\begin{gathered}
-C_{1}+C_{2}|\xi|^{2} \leq a(\xi, y) \cdot \xi,|a(\xi, y)| \leq C_{3}|\xi|+C_{4} \\
|g(u, y)| \leq C_{5}|u|+C_{6} \\
|g(u, y)-g(v, y)| \leq C_{7}|u-v| \\
\left|g_{u}^{\prime}(u, y)-g_{u}^{\prime}(v, y)\right| \leq C_{8}|u-v|(1+|u|+|v|)^{-1}
\end{gathered}
$$

(iv)

$$
\int_{S \cap Y} g(u, y) \mathrm{d} \sigma_{y}=0, \forall u \in \mathbb{R}
$$


Let us rewrite (1.1) in an abstract form. To this end consider the space $X_{\varepsilon}=$ $W^{1,2}\left(\Omega_{\varepsilon}\right)$ and its dual $X_{\varepsilon}^{*}$ with respect to the duality pairing $\langle\cdot, \cdot\rangle_{\varepsilon}$ induced by the standard inner product in $L^{2}\left(\Omega_{\varepsilon}\right)$. Define the operators $\mathcal{A}_{\varepsilon}, \mathcal{G}_{\varepsilon}: X_{\varepsilon} \rightarrow X_{\varepsilon}^{*}$ by

$$
\begin{array}{r}
\left\langle\mathcal{A}_{\varepsilon}(u), v\right\rangle_{\varepsilon}=\int_{\Omega_{\varepsilon}} a(D u, x / \varepsilon) \cdot D v \mathrm{~d} x,\left\langle\mathcal{G}_{\varepsilon}(u), v\right\rangle_{\varepsilon}=\int_{S_{\varepsilon}} g(u, x / \varepsilon) v \mathrm{~d} \sigma \\
\forall v \in X^{\varepsilon}\left(=W^{1,2}\left(\Omega_{\varepsilon}\right)\right) .
\end{array}
$$

In terms of these operators (1.1) reads

$$
\mathcal{A}_{\varepsilon}\left(u_{\varepsilon}\right)+\lambda u_{\varepsilon}-\mathcal{G}_{\varepsilon}\left(u_{\varepsilon}\right)=f
$$

According to the assumptions (i)-(iii) the operator $\mathcal{A}_{\varepsilon}$ is monotone and continuous while $\mathcal{G}_{\varepsilon}$ is a compact operator. It follows that $\mathcal{F}_{\varepsilon}(u)=\mathcal{A}_{\varepsilon}(u)+\lambda u-\mathcal{G}_{\varepsilon}(u)(\lambda>0)$ is a bounded continuous and pseudo-monotone operator (recall that $\mathcal{F}_{\varepsilon}: X_{\varepsilon} \rightarrow X_{\varepsilon}^{*}$ is pseudo-monotone if $u^{(i)} \rightarrow u$ weakly in $X_{\varepsilon}$ and $\lim \sup _{i \rightarrow \infty}\left\langle\mathcal{F}_{\varepsilon}\left(u^{(i)}\right), u^{(i)}-u\right\rangle_{\varepsilon} \leq 0$ $\operatorname{imply}\left\langle\mathcal{F}_{\varepsilon}(u), u-v\right\rangle_{\varepsilon} \leq \liminf _{i \rightarrow \infty}\left\langle\mathcal{F}_{\varepsilon}\left(u^{(i)}\right), u^{(i)}-v\right\rangle_{\varepsilon}$ for all $\left.v \in X_{\varepsilon}\right)$. Then for any $f \in L^{2}(\Omega)$ problem (1.1) has a (possibly not unique) solution $u_{\varepsilon} \in X_{\varepsilon}$ when $\varepsilon \leq \varepsilon_{0}$, $\lambda \geq \lambda_{0}$ (where $\lambda_{0}, \varepsilon_{0}>0$ are specified in Theorem 1 below) by Brezis' theorem (see, e.g., [21], Chapter II), thanks to the following coercivity result

Theorem 1. Under assumptions (i)-(iv) there are $\lambda_{0}, \varepsilon_{0}>0$ such that

$$
\left\langle\mathcal{A}_{\varepsilon} u+\lambda u-\mathcal{G}_{\varepsilon}(u), u\right\rangle_{\varepsilon} \geq \kappa_{1}\|u\|_{X_{\varepsilon}}^{2}-\kappa_{2},
$$

when $\|u\|_{X_{\varepsilon}} \geq R$, for some $\kappa_{1}>0, \kappa_{2}>0$ and $R>0$ independent of $\varepsilon \leq \varepsilon_{0}$ and $\lambda \geq \lambda_{0}$

Under the above assumptions on the perforated domain $\Omega_{\varepsilon}$ there is a bounded linear extension operator $P_{\varepsilon}: W^{1,2}\left(\Omega_{\varepsilon}\right) \rightarrow W^{1,2}(\Omega)\left(P_{\varepsilon} v=v\right.$ in $\Omega_{\varepsilon}$ for any $\left.v \in W^{1,2}\left(\Omega_{\varepsilon}\right)\right)$ and $\left\|P_{\varepsilon} v\right\|_{W^{1,2}(\Omega)} \leq C\|v\|_{W^{1,2}\left(\Omega_{\varepsilon}\right)},\left\|P_{\varepsilon} v\right\|_{L^{2}(\Omega)} \leq C\|v\|_{L^{2}\left(\Omega_{\varepsilon}\right)}$ with $C$ independent of $\varepsilon$ (see,e.g. [2]). We keep the notation $u_{\varepsilon}$ for the solution of (1.1) extended to $\Omega_{\varepsilon}\left(u_{\varepsilon}=P_{\varepsilon} u_{\varepsilon}\right)$ and study the asymptotic behavior of $u_{\varepsilon}$ as $\varepsilon \rightarrow 0$.

The first main result of this work is

Theorem 2. Assume that conditions (i)-(iv) are satisfied and $f$ in (1.1) belongs to $L^{2}(\Omega)$. Let $\lambda_{0}>0$ be as in Theorem 1. Then for any $\lambda \geq \lambda_{0}$, solutions $u_{\varepsilon}$ of 
(1.1) and their derivatives $D u_{\varepsilon}$ two-scale converge as $\varepsilon \rightarrow 0$ (up to extracting a subsequence) to $U_{0}(x)$ and $D U_{0}(x)+D_{y} U_{1}(x, y)$, where the pair $U_{0}(x), U_{1}(x, y)$ is a solution of the two-scale homogenized problem: find $U_{0}(x) \in W^{1,2}(\Omega), U_{1}(x, y) \in$ $L^{2}\left(\Omega ; W_{p e r}^{1,2}(Y)\right)$ such that

$$
\begin{aligned}
\int_{\Omega} \int_{Y^{*}}\left(a\left(D U_{0}+D_{y} U_{1}, y\right) \cdot\left(D \Phi_{0}+D_{y} \Phi_{1}\right) \mathrm{d} y \mathrm{~d} x\right. \\
\quad-\int_{\Omega} \int_{S \cap Y}\left(g\left(U_{0}, y\right) \Phi_{1}(x, y)+g_{u}^{\prime}\left(U_{0}, y\right) \Phi_{0} U_{1}(x, y)\right) \mathrm{d} \sigma_{y} \mathrm{~d} x \\
-\int_{\Omega} \int_{S \cap Y} D_{x}\left(g\left(U_{0}, y\right) \Phi_{0}\right) \cdot y \mathrm{~d} \sigma_{y} \mathrm{~d} x-\int_{\Omega}\left|Y^{*}\right|\left(f-\lambda U_{0}\right) \Phi_{0} \mathrm{~d} x=0
\end{aligned}
$$

for any $\Phi_{0}(x) \in W^{1,2}(\Omega), \Phi_{1}(x, y) \in L^{2}\left(\Omega ; W_{\text {per }}^{1,2}(Y)\right)$. In particular, $u_{\varepsilon}$ converge weakly in $W^{1,2}(\Omega)$ to a solution $U_{0}$ of the homogenized problem (1.3), where $a^{*}(\xi, u)$, $b^{*}(\xi, u), g^{*}(u)$ are defined by

$$
\begin{aligned}
a^{*}(\xi, u) & =\int_{Y^{*}} a\left(\xi+D_{y} w, y\right) \mathrm{d} y, \\
b^{*}(\xi, u) & =\int_{S \cap Y} g_{u}^{\prime}(u, y) w \mathrm{~d} \sigma_{y}, \\
g^{*}(u) & =\int_{Y^{*}} g(u, y) y \mathrm{~d} \sigma_{y},
\end{aligned}
$$

and $w=w(y ; \xi, u)$ is a unique (up to an additive constant) solution of the cell problem

$$
\left\{\begin{array}{l}
\operatorname{div} a\left(\xi+D_{y} w, y\right)=0 \text { in } Y^{*} \\
a\left(\xi+D_{y} w, y\right) \cdot \nu=g(u, y) \text { on } S \cap Y \\
w \text { is } Y \text {-periodic. }
\end{array}\right.
$$

Remark 3. Note that (2.9) defines $U_{1}(x, y)$ modulo an arbitrary function $\tilde{U}_{1}(x, y) \in$ $L^{2}\left(\Omega, W_{\text {per }}^{1,2}(Y)\right.$ such that $U_{1}(x, y)=0$ for $y \in Y^{*}$. This is due to the freedom in the particular choice of the extension operators $P_{\varepsilon}$.

Remark 4. The third term in (2.9) is reduced by integrating by parts to the boundary integral

$$
\int_{\Omega} \int_{S \cap Y} D_{x}\left(g\left(U_{0}, y\right) \Phi_{0}\right) \cdot y \mathrm{~d} \sigma_{y} \mathrm{~d} x=\int_{\partial \Omega} \Phi_{0} g^{*}\left(U_{0}\right) \cdot \nu \mathrm{d} \sigma_{x}
$$

that leads to the boundary condition in (1.3) . 
Remark 5. In the linear case, that is when $a$ and $g$ are given by $a(\xi, y)=A(y) \xi$, $g(u, y)=\alpha(y)+u \beta(y)$, the cell problem (2.13) for $w$ splits into three cell problems for $w^{(1)}$,

$$
\left\{\begin{array}{l}
\operatorname{div}\left(A(y)\left(\xi+D_{y} w^{(1)}\right)\right)=0 \text { in } Y^{*} \\
A(y) D_{y} w^{(1)} \cdot \nu=-A(y) \xi \cdot \nu \text { on } S \cap Y \\
w^{(1)} \text { is } Y \text {-periodic, }
\end{array}\right.
$$

and $w^{(k)}(k=2,3)$,

$$
\left\{\begin{array}{l}
\operatorname{div}\left(A(y) D_{y} w^{(k)}\right)=0 \text { in } Y^{*} \\
A(y) D_{y} w^{(k)} \cdot \nu=\delta_{2 k} \beta(y)+\delta_{3 k} \alpha(y) \text { on } S \cap Y \\
w^{(2)} \text { is } Y \text {-periodic, }
\end{array}\right.
$$

$\left(\delta_{i j}\right.$ is the Kronecker delta) so that $w=w^{(1)}+u w^{(2)}+w^{(3)}$. Then the homogenized equation takes form

$$
\operatorname{div} A^{\text {hom }} D U_{0}+B^{\text {hom }} \cdot D U_{0}+C^{\text {hom }} U_{0}+D^{\text {hom }}+\left|Y^{*}\right|\left(f-\lambda U_{0}\right)=0,
$$

where the homogenized matrix $A^{\text {hom }}$ coincides with the classical effective matrix for the Neumann problem in perforated domains,

$$
A^{\text {hom }} \xi=\int_{Y^{*}} A(y)\left(\xi+D_{y} w^{(1)}\right) \mathrm{d} y
$$

and

$$
\begin{gathered}
B^{\mathrm{hom}} \cdot \xi=\int_{Y^{*}} A(y) D_{y} w^{(2)} \cdot\left(\xi+D_{y} w^{(1)}\right) \mathrm{d} y \\
C^{\mathrm{hom}}=\int_{Y^{*}} A(y) D_{y} w^{(2)} \cdot D_{y} w^{(2)} \mathrm{d} y, D^{\mathrm{hom}}=\int_{Y^{*}} A(y) D_{y} w^{(2)} \cdot D_{y} w^{(3)} \mathrm{d} y .
\end{gathered}
$$

Note, that $B^{\text {hom }}=0$ in the selfadjoint case (when $A=A^{T}$ ).

In the case of the parabolic problem (1.2) we prove that there is a unique solution $u_{\varepsilon}$ and its asymptotic behavior in the leading term is described by the homogenized problem (1.4). Formulating the convergence result we assume as before $u_{\varepsilon}$ extended onto the whole domain $\Omega$ by means of the extension operator $P_{\varepsilon}$

Theorem 6. Assume that conditions (i) - (iv) are satisfied. Then, if $f \in L^{2}((0, T) \times$ $\Omega$ ) and $\tilde{u} \in L^{2}(\Omega)$, there is a unique solution of problem (1.2) and it converges weakly 
in $L^{2}\left(0, T ; W^{1,2}(\Omega)\right)$ as $\varepsilon \rightarrow 0$ (up to extracting a subsequence) to a solution $U_{0}$ of the homogenized problem (1.4), where $a^{*}, b^{*}, g^{*}$ are defined by (2.10), (2.11), (2.12), (2.13).

\section{Proof of the convergence result for the station- ary problem}

It follows from Theorem 1 that $\left\|u_{\varepsilon}\right\|_{W^{1,2}(\Omega)} \leq C$, where $C$ is independent of $\varepsilon$. Therefore, up to extracting a subsequence,

$$
\begin{gathered}
u_{\varepsilon} \rightarrow U_{0}(x) \text { two-scale } \\
D u_{\varepsilon} \rightarrow D U_{0}(x)+D_{y} U_{1}(x, y) \text { two-scale. }
\end{gathered}
$$

Show that the pair $\left(U_{0}, U_{1}\right)$ solves (2.9) . To this end we chose arbitrary functions $V_{0}(x) \in C^{\infty}(\bar{\Omega}), V_{1}(x, y) \in C^{\infty}(\bar{\Omega} \times \bar{Y})$ with $V_{1}(x, y)$ being $Y$-periodic in $y$, set $v_{\varepsilon}=V_{0}(x)+\varepsilon V_{1}(x, x / \varepsilon)$, and substitute the test function $w_{\varepsilon}=u_{\varepsilon}-v_{\varepsilon}$ in the weak formulation of (1.1),

$$
\int_{\Omega_{\varepsilon}}\left(a\left(D u_{\varepsilon}, x / \varepsilon\right) \cdot D w_{\varepsilon}+\lambda u_{\varepsilon} w_{\varepsilon}\right) \mathrm{d} x-\int_{S_{\varepsilon}} g\left(u_{\varepsilon}, x / \varepsilon\right) w_{\varepsilon} \mathrm{d} \sigma=\int_{\Omega_{\varepsilon}} f w_{\varepsilon} \mathrm{d} x .
$$

In view of the monotonicity assumption (2.1) we then have from (3.3),

$$
\begin{aligned}
& \int_{\Omega_{\varepsilon}}\left(a\left(D v_{\varepsilon}, x / \varepsilon\right) \cdot D\left(u_{\varepsilon}-v_{\varepsilon}\right)+\lambda v_{\varepsilon}\left(u_{\varepsilon}-v_{\varepsilon}\right)\right) \mathrm{d} x-\int_{S_{\varepsilon}} g\left(u_{\varepsilon}, x / \varepsilon\right)\left(u_{\varepsilon}-v_{\varepsilon}\right) \mathrm{d} \sigma \\
& -\int_{\Omega_{\varepsilon}} f\left(u_{\varepsilon}-v_{\varepsilon}\right) \mathrm{d} x \leq 0 .
\end{aligned}
$$

Since $D v_{\varepsilon}=D V_{0}(x)+D_{y} V_{1}(x, x / \varepsilon)+\varepsilon D_{x} V_{1}(x, x / \varepsilon)$, by using (i) and (2.2) one easily shows that $\chi_{\varepsilon} a\left(D v_{\varepsilon}, x / \varepsilon\right) \rightarrow \chi(y) a\left(D V_{0}(x)+D_{y} V_{1}(x, y), y\right)$ in the strong two-scale sense, where $\chi_{\varepsilon}, \chi$ are the characteristic functions of $\Omega_{\varepsilon}$ and $Y^{*}$, respectively. This allows to pass to the limit in the first term of l.h.s. of (3.4 to get

$$
\begin{aligned}
& \int_{\Omega_{\varepsilon}}\left(a\left(D v_{\varepsilon}, x / \varepsilon\right) \cdot D\left(u_{\varepsilon}-v_{\varepsilon}\right)+\lambda v_{\varepsilon}\left(u_{\varepsilon}-v_{\varepsilon}\right)\right) \mathrm{d} x \rightarrow \\
& \int_{\Omega}\left(\int_{Y^{*}}\left(a\left(D V_{0}+D_{y} V_{1}, y\right) \cdot\left(D U_{0}+D_{y} U_{1}-D V_{0}-D_{y} V_{1}\right)+\lambda V_{0}\left(U_{0}-V_{0}\right)\right) \mathrm{d} y\right) \mathrm{d} x
\end{aligned}
$$


also, the limit transition in the last term in l.h.s. of (3.4) yields

$$
\int_{\Omega_{\varepsilon}} f\left(u_{\varepsilon}-v_{\varepsilon}\right) \mathrm{d} x \rightarrow \int_{\Omega}\left(\int_{Y^{*}} f\left(U_{0}-V_{0}\right) \mathrm{d} y\right) \mathrm{d} x .
$$

Finally, passing to the limit in the middle term we get

$$
\begin{aligned}
& \int_{S_{\varepsilon}} g\left(u_{\varepsilon}, x / \varepsilon\right)\left(u_{\varepsilon}-v_{\varepsilon}\right) \mathrm{d} \sigma \rightarrow \\
& \int_{\Omega}\left(\int_{S \cap Y} g\left(U_{0}, y\right)\left(D\left(U_{0}-V_{0}\right) \cdot y+U_{1}(x, y)-V_{1}(x, y)\right) \mathrm{d} \sigma_{y}\right) \mathrm{d} x \\
& \quad+\int_{\Omega}\left(\int_{S \cap Y} g_{u}^{\prime}\left(U_{0}, y\right)\left(U_{0}-V_{0}\right)\left(D U_{0} \cdot y+U_{1}(x, y)\right) \mathrm{d} \sigma_{y}\right) \mathrm{d} x .
\end{aligned}
$$

The most nontrivial point is to obtain (3.7). The proof of (3.7) is presented in full details through Sections 4, 5 and is based on the following result, which is of an interest itself,

Proposition 7. Assume that $q(x, y) \in C\left(\Omega ; E^{\infty}(S)\right)$ satisfies

(a) $\left|q(x, y)-q\left(x^{\prime}, y\right)\right| \leq C\left|x-x^{\prime}\right|$ with $C>0$ independent of $x, x^{\prime} \in \Omega$ and $y \in S$;

(b) $q(x, y)$ is $Y$-periodic in $y \in S$;

(c) $\int_{Y \cap S} q(x, y) \mathrm{d} \sigma_{y}=0$ for all $x \in \Omega$,

then for any sequence $w_{\varepsilon} \in W^{1,2}(\Omega)$ such that

$$
w_{\varepsilon}(x) \rightarrow W_{0}(x), D w_{\varepsilon}(x) \rightarrow D W_{0}(x)+D_{y} W_{1}(x, y) \text { two scale as } \varepsilon \rightarrow 0 .
$$

we have

$$
\int_{S_{\varepsilon}} q(x, x / \varepsilon)\left(w_{\varepsilon}-\bar{w}_{\varepsilon}\right) \mathrm{d} \sigma \rightarrow \int_{\Omega} \int_{Y \cap S} q(x, y)\left(D W_{0} \cdot y+W_{1}(x, y)\right) \mathrm{d} \sigma_{y} \mathrm{~d} x .
$$

Here and in what follows we use the notation $\bar{w}_{\varepsilon}$ for the piecewise constant function obtained by averaging over the cells $Y_{\varepsilon}^{(m)}$,

$$
\bar{w}_{\varepsilon}(x)=\frac{1}{\varepsilon^{N}} \int_{Y_{\varepsilon}^{(m)}} w_{\varepsilon}(y) \mathrm{d} y, \text { for } x \in Y_{\varepsilon}^{(m)} .
$$


Thus (3.4)-(3.7) yield

$$
\begin{gathered}
\int_{\Omega}\left(\int_{Y^{*}}\left(a\left(D V_{0}+D_{y} V_{1}, y\right) \cdot\left(D U_{0}+D_{y} U_{1}-D V_{0}-D_{y} V_{1}\right)+\lambda V_{0}\left(U_{0}-V_{0}\right)\right) \mathrm{d} y\right) \mathrm{d} x \\
-\int_{\Omega}\left(\int_{S \cap Y} g\left(U_{0}, y\right)\left(D\left(U_{0}-V_{0}\right) \cdot y+U_{1}(x, y)-V_{1}(x, y)\right) \mathrm{d} \sigma_{y}\right) \mathrm{d} x \\
-\int_{\Omega}\left(\int_{S \cap Y} g_{u}^{\prime}\left(U_{0}, y\right)\left(U_{0}-V_{0}\right)\left(D U_{0} \cdot y+U_{1}(x, y)\right) \mathrm{d} \sigma_{y}\right) \mathrm{d} x \\
-\int_{\Omega}\left(\int_{Y^{*}} f\left(U_{0}-V_{0}\right) \mathrm{d} y\right) \mathrm{d} x \leq 0, \quad(3.11)
\end{gathered}
$$

By an approximation argument, using (i)-(iv) we see that (3.11) holds for any $V_{0} \in$ $W^{1,2}(\Omega)$ and $V_{1} \in L^{2}\left(\Omega ; W_{p e r}^{1,2}(Y)\right)$. Now, choosing $V_{0}=U_{0} \pm \tau \Phi_{0}, V_{1}=U_{1} \pm \tau \Phi_{1}$, $(\tau>0)$, dividing (3.11) by $\tau$ and passing to the limit as $\tau \rightarrow 0$, we obtain the two-scale homogenization problem (2.9).

Let us clarify details in the final part of the above proof when passing from smooth $V_{0}$ and $V_{1}$ to arbitrary functions $V_{0} \in W^{1,2}(\Omega)$ and $V_{1} \in L^{2}\left(\Omega ; W_{p e r}^{1,2}(Y)\right)$ in (3.11). For the for the first term in the l.h.s. this transition is justified by Nemytskii's theorem (see, e.g., [21], Chapter II); and it is a trivial task for the last term. The second and third terms, corresponding to the limiting functional $M\left(U_{0}, U_{1}, V_{0}, V_{1}\right)$ in (3.7), require more attention. Let us rewrite $M\left(U_{0}, U_{1}, V_{0}, V_{1}\right)$ as

$$
\begin{array}{r}
M\left(U_{0}, U_{1}, V_{0}, V_{1}\right)=\int_{\Omega}\left(g^{*}\left(U_{0}\right) \cdot D\left(U_{0}-V_{0}\right)+\left(U_{0}-V_{0}\right)\left(g^{*}\right)^{\prime}\left(U_{0}\right) \cdot D U_{0}\right) \mathrm{d} x \\
\quad+\int_{\Omega} \int_{Y^{*}} D_{y} \Theta\left(y ; U_{0}\right) \cdot D_{y}\left(U_{1}(x, y)-V_{1}(x, y)\right) \mathrm{d} y \mathrm{~d} x \\
\quad+\int_{\Omega} \int_{Y^{*}}\left(U_{0}-V_{0}\right) D_{y} \Theta_{u}^{\prime}\left(y ; U_{0}\right) \cdot D_{y} U_{1}(x, y) \mathrm{d} y \mathrm{~d} x,
\end{array}
$$

where $\left(g^{*}\right)^{\prime}$ denotes the derivative of $g^{*}$, and $\Theta(y ; u)$ is a solution of the problem

$$
\left\{\begin{array}{l}
\Delta_{y} \Theta=0 \text { in } Y^{*} \\
\frac{\partial \Theta}{\partial \nu}=g(u, y) \text { on } S \cap Y \\
\Theta \text { is } Y \text {-periodic. }
\end{array}\right.
$$

It follows from the assumptions (iii), (iv) that (3.13) has a unique (modulo an additive constant) solution $\Theta(y ; u)$, and $\Theta$ depends regularly on the parameter $u$, more precisely,

$$
\left\|D_{y} \Theta(\cdot ; u)\right\|_{L^{2}\left(Y^{*}\right)} \leq C(|u|+1),
$$




$$
\begin{gathered}
\left\|D_{y} \Theta(\cdot ; u)-D_{y} \Theta(\cdot ; v)\right\|_{L^{2}\left(Y^{*}\right)} \leq C|u-v|, \\
\left\|D_{y} \Theta_{u}^{\prime}(\cdot ; u)-D_{y} \Theta_{u}^{\prime}(\cdot ; v)\right\|_{L^{2}\left(Y^{*}\right)} \leq C|u-v|(1+|u|+|v|)^{-1},
\end{gathered}
$$

where $C$ does not depend on $u, v$. All these properties are demonstrated similarly, e.g., we show (3.14) by using (2.3), (2.6) and the Poincaré inequality (17.6) in $W_{p e r}^{1,2}\left(Y^{*}\right)$ (see Sec. [6),

$$
\left|\int_{Y^{*}} D_{y} \Theta \cdot D_{y} \Theta \mathrm{d} y\right|=\left|\int_{S \cap Y} g(u, y)\left(\Theta-\frac{1}{\left|Y^{*}\right|} \int_{Y^{*}} \Theta \mathrm{d} y\right) \mathrm{d} y\right| \leq C(|u|+1)\left\|D_{y} \Theta\right\|_{L^{2}\left(Y^{*}\right)} .
$$

The bounds (3.14) - (3.16) in conjunction with assumptions (2.3) - (2.5) imply

Proposition 8. The functional $M\left(U_{0}, U_{1}, V_{0}, V_{1}\right)$ defined by (3.12) (or, equivalently, by the r.h.s. of (3.7)) is continuous in $W^{1,2}(\Omega) \times L^{2}\left(\Omega ; W_{\text {per }}^{1,2}\left(Y^{*}\right)\right) \times W^{1,2}(\Omega) \times$ $L^{2}\left(\Omega ; W_{p e r}^{1,2}\left(Y^{*}\right)\right)$.

\section{Auxiliary results and proof of Theorem 1}

$\mathbf{1}$ (Some inequalities). Recall the classical inequalities in Sobolev spaces,

$$
\begin{aligned}
& \int_{S \cap Y}\left|v-\int_{Y} v \mathrm{~d} x\right|^{2} \mathrm{~d} \sigma \leq C \int_{Y}|D v|^{2} \mathrm{~d} x, \forall v \in W^{1,2}(Y) \text { (the Poincaré inequality), } \\
& \int_{S \cap Y}|v|^{2} \mathrm{~d} \sigma \leq C \int_{Y}\left(|v|^{2}+|D v|^{2}\right) \mathrm{d} x, \forall v \in W^{1,2}(Y) \text { (the trace inequality). }
\end{aligned}
$$

By an easy scaling argument (4.1), (4.2) lead to the inequalities

$$
\begin{gathered}
\int_{S_{\varepsilon}}\left|v_{\varepsilon}-\bar{v}_{\varepsilon}\right|^{2} \mathrm{~d} \sigma \leq C \varepsilon \int_{\Omega}\left|D v_{\varepsilon}\right|^{2} \mathrm{~d} x, \\
\int_{S_{\varepsilon}}\left|v_{\varepsilon}\right|^{2} \mathrm{~d} \sigma \leq C \varepsilon^{-1}\left(\int_{\Omega}\left|v_{\varepsilon}\right|^{2} \mathrm{~d} x+\varepsilon^{2} \int_{\Omega}\left|D v_{\varepsilon}\right|^{2} \mathrm{~d} x\right),
\end{gathered}
$$

for any $v_{\varepsilon} \in W^{1,2}(\Omega)$, where $\bar{v}_{\varepsilon}$ stands for piecewise constant function obtained by averaging over each cell $Y_{\varepsilon}^{(m)}$ (cf. (3.10) ), and $C$ depends only on $S$. We also will make use of the following inequality, which is a simple consequence of Jensen's inequality, for any $r \geq 1$,

$$
\int_{S_{\varepsilon}}\left|\bar{v}_{\varepsilon}\right|^{r} \mathrm{~d} \sigma \leq C \varepsilon^{-1} \int_{\Omega}\left|v_{\varepsilon}\right|^{r} \mathrm{~d} x
$$


where $C>0$ is independent of $r$ and $v_{\varepsilon}$.

2(An asymptotic representation for surface integral in (3.4). To pass to the limit as $\varepsilon \rightarrow 0$ in the surface integral in (3.4) we use

Lemma 9. Let $u_{\varepsilon}, w_{\varepsilon} \in W^{1,2}(\Omega)$, then

$$
\begin{aligned}
\int_{S_{\varepsilon}} g\left(u_{\varepsilon}, x / \varepsilon\right) w_{\varepsilon} \mathrm{d} x=\int_{S_{\varepsilon}} g\left(\bar{u}_{\varepsilon}, x / \varepsilon\right)\left(w_{\varepsilon}\right. & \left.-\bar{w}_{\varepsilon}\right) \mathrm{d} \sigma \\
& +\int_{S_{\varepsilon}} g_{u}^{\prime}\left(\bar{u}_{\varepsilon}, x / \varepsilon\right) \bar{w}_{\varepsilon}\left(u_{\varepsilon}-\bar{u}_{\varepsilon}\right) \mathrm{d} \sigma+\varrho_{\varepsilon}
\end{aligned}
$$

and

$$
\left|\varrho_{\varepsilon}\right| \leq C\left(\varepsilon+\left(\varepsilon\left\|w_{\varepsilon}\right\|_{L^{2}(\Omega)}\right)^{2 /(N+2)}\right)\left(\left\|w_{\varepsilon}\right\|_{W^{1,2}(\Omega)}^{2}+\left\|u_{\varepsilon}\right\|_{W^{1,2}(\Omega)}^{2}\right) .
$$

Proof. We have,

$$
\begin{aligned}
g\left(u_{\varepsilon}, x / \varepsilon\right) w_{\varepsilon}=g\left(\bar{u}_{\varepsilon}, x / \varepsilon\right)\left(w_{\varepsilon}-\bar{w}_{\varepsilon}\right) & +\left(g\left(u_{\varepsilon}, x / \varepsilon\right)-\left(g\left(\bar{u}_{\varepsilon}, x / \varepsilon\right)\right)\left(w_{\varepsilon}-\bar{w}_{\varepsilon}\right)\right. \\
& +\left(g\left(u_{\varepsilon}, x / \varepsilon\right)-g\left(\bar{u}_{\varepsilon}, x / \varepsilon\right)\right) \bar{w}_{\varepsilon}+g\left(\bar{u}_{\varepsilon}, x / \varepsilon\right) \bar{w}_{\varepsilon}
\end{aligned}
$$

therefore (in view of (2.6))

$$
\begin{aligned}
\int_{S_{\varepsilon}} g\left(u_{\varepsilon}, x / \varepsilon\right) w_{\varepsilon} \mathrm{d} \sigma & =\int_{S_{\varepsilon}} g\left(\bar{u}_{\varepsilon}, x / \varepsilon\right)\left(w_{\varepsilon}-\bar{w}_{\varepsilon}\right) \mathrm{d} \sigma \\
& +\int_{S_{\varepsilon}}\left(g\left(u_{\varepsilon}, x / \varepsilon\right)-\left(g\left(\bar{u}_{\varepsilon}, x / \varepsilon\right)\right)\left(w_{\varepsilon}-\bar{w}_{\varepsilon}\right) \mathrm{d} \sigma\right. \\
& +\int_{S_{\varepsilon}}\left(g\left(u_{\varepsilon}, x / \varepsilon\right)-g\left(\bar{u}_{\varepsilon}, x / \varepsilon\right)\right) \bar{w}_{\varepsilon} \mathrm{d} \sigma=I_{1}+I_{2}+I_{3} .
\end{aligned}
$$

The term $I_{2}$ gives vanishing contribution when $\varepsilon \rightarrow 0$. Really, by (2.4) and (4.3),

$$
\left|I_{2}\right| \leq C \int_{S_{\varepsilon}}\left|u_{\varepsilon}-\bar{u}_{\varepsilon}\right|\left|w_{\varepsilon}-\bar{w}_{\varepsilon}\right| \mathrm{d} \sigma \leq C \varepsilon\left\|D u_{\varepsilon}\right\|_{L^{2}(\Omega)}\left\|D w_{\varepsilon}\right\|_{L^{2}(\Omega)} .
$$

The term $I_{3}$ can be written as

$$
\begin{aligned}
I_{3}=\int_{0}^{1} \mathrm{~d} t & \int_{S_{\varepsilon}}\left(g_{u}^{\prime}\left(\bar{u}_{\varepsilon}+t\left(u_{\varepsilon}-\bar{u}_{\varepsilon}\right), x / \varepsilon\right)-g_{u}^{\prime}\left(\bar{u}_{\varepsilon}, x / \varepsilon\right)\right) \bar{w}_{\varepsilon}\left(u_{\varepsilon}-\bar{u}_{\varepsilon}\right) \mathrm{d} \sigma \\
& \quad+\int_{S_{\varepsilon}} g_{u}^{\prime}\left(\bar{u}_{\varepsilon}, x / \varepsilon\right) \bar{w}_{\varepsilon}\left(u_{\varepsilon}-\bar{u}_{\varepsilon}\right) \mathrm{d} \sigma=\tilde{I}_{3}+\int_{S_{\varepsilon}} g_{u}^{\prime}\left(\bar{u}_{\varepsilon}, x / \varepsilon\right) \bar{w}_{\varepsilon}\left(u_{\varepsilon}-\bar{u}_{\varepsilon}\right) \mathrm{d} \sigma
\end{aligned}
$$


By using (2.5) we get

$$
\left|\tilde{I}_{3}\right| \leq C \sup _{0 \leq t \leq 1} \int_{S_{\varepsilon}} \frac{t\left|u_{\varepsilon}-\bar{u}_{\varepsilon}\right|^{2}\left|\bar{w}_{\varepsilon}\right|}{1+\left|\bar{u}_{\varepsilon}\right|+\left|\bar{u}_{\varepsilon}+t\left(u_{\varepsilon}-\bar{u}_{\varepsilon}\right)\right|} \mathrm{d} \sigma
$$

which yields after applying the Holder inequality,

$$
\begin{aligned}
\left|\tilde{I}_{3}\right| \leq C \sup _{0 \leq t \leq 1} \int_{S_{\varepsilon}} \frac{t\left|u_{\varepsilon}-\bar{u}_{\varepsilon}\right|^{2}\left|\bar{w}_{\varepsilon}\right|}{1+t\left|u_{\varepsilon}-\bar{u}_{\varepsilon}\right|} \mathrm{d} \sigma \leq C\left(\int_{S_{\varepsilon}}\left|\bar{w}_{\varepsilon}\right|^{q} \mathrm{~d} \sigma\right)^{1 / q} \\
\times \sup _{0 \leq t \leq 1}\left(\int_{S_{\varepsilon}}\left|u_{\varepsilon}-\bar{u}_{\varepsilon}\right|^{2} \frac{t^{q^{\prime}}\left|u_{\varepsilon}-\bar{u}_{\varepsilon}\right|^{2 q^{\prime}-2}}{\left(1+t\left|u_{\varepsilon}-\bar{u}_{\varepsilon}\right|\right)^{\prime}} \mathrm{d} \sigma\right)^{1 / q^{\prime}},
\end{aligned}
$$

where $q^{\prime}=q /(q-1)$ and $q=2(N+2) / N$. Note that the embedding $W^{1,2}(\Omega) \subset L^{q}(\Omega)$ is compact, moreover one has (see, e.g., [12])

$$
\exists C>0 \text { such that }\|u\|_{L^{q}(\Omega)} \leq C\|u\|_{W^{1,2}(\Omega)}^{2 / q}\|u\|_{L^{2}(\Omega)}^{4 /(N q)} \forall u \in W^{1,2}(\Omega) .
$$

Since $1<q^{\prime}<2$, we have

$$
\frac{t^{q^{\prime}}\left|u_{\varepsilon}-\bar{u}_{\varepsilon}\right|^{2 q^{\prime}-2}}{\left(1+t\left|u_{\varepsilon}-\bar{u}_{\varepsilon}\right|\right)^{q^{\prime}}} \leq \frac{t^{2 q^{\prime}-2}\left|u_{\varepsilon}-\bar{u}_{\varepsilon}\right|^{2 q^{\prime}-2}}{\left(1+t\left|u_{\varepsilon}-\bar{u}_{\varepsilon}\right|\right)^{2 q^{\prime}-2}} \frac{t^{2-q^{\prime}}}{\left(1+t\left|u_{\varepsilon}-\bar{u}_{\varepsilon}\right|\right)^{2-q^{\prime}}} \leq 1
$$

for any $0 \leq t \leq 1$. Therefore, by using (4.3), (4.5) and (4.9) we get

$$
\begin{aligned}
& \left|\tilde{I}_{3}\right| \leq C \varepsilon^{-1 / q-1 / q^{\prime}+2 / q^{\prime}}\left\|w_{\varepsilon}\right\|_{L^{q}(\Omega)}\left\|D u_{\varepsilon}\right\|_{L^{2}(\Omega)}^{2 / q^{\prime}} \\
& \leq C \varepsilon^{2 /(N+2)}\left\|w_{\varepsilon}\right\|_{W^{1,2}(\Omega)}^{2 / q}\left\|w_{\varepsilon}\right\|_{L^{2}(\Omega)}^{4 /(N q)}\left\|D u_{\varepsilon}\right\|_{L^{2}(\Omega)}^{2 / q^{\prime}} \\
& \quad \leq C\left(\varepsilon\left\|w_{\varepsilon}\right\|_{L^{2}(\Omega)}\right)^{2 /(N+2)}\left(\left\|w_{\varepsilon}\right\|_{W^{1,2}(\Omega)}^{2}+\left\|D u_{\varepsilon}\right\|_{L^{2}(\Omega)}^{2}\right)
\end{aligned}
$$

where we have used also the Young inequality. Bounds (4.10) and (4.10) yield (4.7) (since $\left|\varrho_{\varepsilon}\right| \leq\left|I_{2}\right|+\left|\tilde{I}_{3}\right|$ ). Lemma is proved.

The proof of the next technical result is similar to Lemma 9 (and left to the reader).

Lemma 10. If $u_{\varepsilon}, u_{\varepsilon}^{(1)} \in W^{1,2}(\Omega), v_{\varepsilon} \in L^{\infty}(\Omega) \cap W^{1,2}(\Omega)$, then setting $w_{\varepsilon}=u_{\varepsilon}-u_{\varepsilon}^{(1)}$ we have

$$
\begin{gathered}
\left|\int_{S_{\varepsilon}}\left(g\left(\bar{u}_{\varepsilon}, x / \varepsilon\right)-g\left(\bar{u}_{\varepsilon}^{(1)}, x / \varepsilon\right)\right)\left(u_{\varepsilon}-v_{\varepsilon}-\bar{u}_{\varepsilon}+\bar{v}_{\varepsilon}\right) \mathrm{d} \sigma\right| \leq C\left\|w_{\varepsilon}\right\|_{L^{2}(\Omega)}\left\|D\left(u_{\varepsilon}-v_{\varepsilon}\right)\right\|_{L^{2}(\Omega)}, \\
\left|\int_{S_{\varepsilon}}\left(g_{u}^{\prime}\left(\bar{u}_{\varepsilon}, x / \varepsilon\right) \bar{u}_{\varepsilon}-g_{u}^{\prime}\left(\bar{u}_{\varepsilon}^{(1)}, x / \varepsilon\right) \bar{u}_{\varepsilon}^{(1)}\right)\left(u_{\varepsilon}-\bar{u}_{\varepsilon}\right) \mathrm{d} \sigma\right| \leq C\left\|w_{\varepsilon}\right\|_{L^{2}(\Omega)}\left\|D u_{\varepsilon}\right\|_{L^{2}(\Omega)}, \\
\left.\mid \int_{S_{\varepsilon}}\left(g_{u}^{\prime}\left(\bar{u}_{\varepsilon}, x / \varepsilon\right)-g_{u}^{\prime}\left(\bar{u}_{\varepsilon}^{(1)}, x / \varepsilon\right)\right)\right) \bar{v}_{\varepsilon}\left(u_{\varepsilon}-\bar{u}_{\varepsilon}\right) \mathrm{d} \sigma \mid \leq C\left\|w_{\varepsilon}\right\|_{L^{2}(\Omega)}\|v\|_{L^{\infty}(\Omega)}\left\|D u_{\varepsilon}\right\|_{L^{2}(\Omega)} .
\end{gathered}
$$


3(Proof of Theorem 1). Assume by contradiction that there are sequences $\varepsilon_{k} \rightarrow 0$, $\lambda_{k} \rightarrow+\infty$ and $u_{k} \in W^{1,2}\left(\Omega_{\varepsilon_{k}}\right)$ such that $\left\|u_{k}\right\|_{X_{\varepsilon_{k}}} \rightarrow \infty$,

$$
\left\langle\mathcal{A}_{\varepsilon_{k}}\left(u_{k}\right), u_{k}\right\rangle_{\varepsilon_{k}}+\lambda_{k}\left\langle u_{k}, u_{k}\right\rangle_{\varepsilon_{k}}-\left\langle\mathcal{G}_{\varepsilon}\left(u_{k}\right), u_{k}\right\rangle_{\varepsilon_{k}} \leq \delta_{k}\left\|u_{k}\right\|_{X_{\varepsilon_{k}}}^{2}
$$

and $\delta_{k} \rightarrow 0$. In view of the definition of $\mathcal{A}_{\varepsilon}$ and $\mathcal{G}_{\varepsilon}$ this implies that

$$
\int_{\Omega_{\varepsilon_{k}}}\left(a\left(D v_{k}, x / \varepsilon\right) \cdot D v_{k}+\lambda_{k}\left|v_{k}\right|^{2}\right) \mathrm{d} x \leq \int_{S_{\varepsilon}} g\left(v_{k}, x / \varepsilon\right) v_{k} \mathrm{~d} \sigma+\delta_{k}\left\|v_{k}\right\|_{W^{1,2}(\Omega)}^{2} \mathrm{~d} x,
$$

where $v_{k}=P_{\varepsilon_{k}} u_{k}$ is the extension of $u_{k}$ onto $\Omega$. By using (2.2) and the properties of the extension operator $P_{\varepsilon}$ we then get, setting $w_{k}=v_{k} /\left\|v_{k}\right\|_{W^{1,2}(\Omega)}$,

$$
\gamma \int_{\Omega}\left|D w_{k}\right|^{2} \mathrm{~d} x+\lambda_{k} \int_{\Omega_{\varepsilon_{k}}}\left|w_{k}\right|^{2} \mathrm{~d} x \leq \frac{1}{\left\|v_{k}\right\|_{W^{1,2}(\Omega)}} \int_{S_{\varepsilon_{k}}} g\left(v_{k}, x / \varepsilon\right) w_{k} \mathrm{~d} \sigma+\tilde{\delta}_{k},
$$

with some $\gamma>0$, where $\tilde{\delta}_{k}=\delta_{k}+C /\left\|v_{k}\right\|_{W^{1,2}(\Omega)}^{2} \rightarrow 0$. Now write

$$
\begin{aligned}
\int_{S_{\varepsilon_{k}}} g\left(v_{k}, x / \varepsilon_{k}\right) w_{k} \mathrm{~d} \sigma=\int_{S_{\varepsilon_{k}}}\left(g\left(v_{k}, x / \varepsilon_{k}\right)-g\left(\bar{v}_{k}, x / \varepsilon_{k}\right)\right) w_{k} \mathrm{~d} \sigma \\
\quad+\int_{S_{\varepsilon_{k}}} g\left(\bar{v}_{k}, x / \varepsilon_{k}\right)\left(w-\bar{w}_{k}\right) \mathrm{d} \sigma=I_{1}+I_{2},
\end{aligned}
$$

where we have used (2.6). We have, by (2.4) and (4.3),

$$
\begin{aligned}
\left|I_{1}\right| \leq C \int_{S_{\varepsilon_{k}}}\left|v_{k}-\bar{v}_{k}\right|\left|w_{k}\right| \mathrm{d} \sigma \leq & C \varepsilon_{k}^{1 / 2}\left(\int_{\Omega}\left|D v_{k}\right|^{2} \mathrm{~d} x\right)^{1 / 2}\left(\int_{S_{\varepsilon_{k}}}\left|w_{k}\right|^{2} \mathrm{~d} x\right)^{1 / 2} \\
& \leq C\left\|D v_{k}\right\|_{L^{2}(\Omega)}\left(\left\|w_{k}\right\|_{L^{2}(\Omega)}+\varepsilon_{k}\left\|D w_{k}\right\|_{L^{2}(\Omega)}\right)
\end{aligned}
$$

Similarly, by (2.3) and (4.5),

$$
\left|I_{2}\right| \leq C \int_{S_{\varepsilon_{k}}}\left|w_{k}-\bar{w}_{k}\right|\left(\left|\bar{v}_{k}\right|+1\right) \mathrm{d} \sigma \leq C\left\|D w_{k}\right\|_{L^{2}(\Omega)}\left(\left\|v_{k}\right\|_{L^{2}(\Omega)}+1\right) .
$$

Thus

$$
\gamma\left\|D w_{k}\right\|_{L^{2}(\Omega)}^{2}+\lambda_{k}\left\|w_{k}\right\|_{L^{2}\left(\Omega_{\varepsilon_{k}}\right)}^{2} \leq C\left(\left\|w_{k}\right\|_{L^{2}(\Omega)}+\varepsilon_{k}\right)+\tilde{\delta}_{k},
$$

where we have used the fact that $\left\|w_{k}\right\|_{W^{1,2}(\Omega)}=1$. Therefore $\left\|w_{k}\right\|_{L^{2}\left(\Omega_{\varepsilon_{k}}\right)}^{2} \rightarrow 0$.

Due to the compactness of the embedding $W^{1,2}(\Omega) \subset L^{2}(\Omega)$, up to a subsequence, $w_{k} \rightarrow w$ strongly in $L^{2}(\Omega)$. On the other hand, according to the structure of perforated domains $\Omega_{\varepsilon}$,

$$
\int_{\Omega_{\varepsilon_{k}}} w_{k} v \mathrm{~d} x \rightarrow\left|Y^{*}\right| \int_{\Omega} w v \mathrm{~d} x \text { for any } v \in L^{2}(\Omega) .
$$


By taking $v=w$ we get $w=0$ (since $\left\|w_{k}\right\|_{L^{2}\left(\Omega_{\varepsilon_{k}}\right)} \rightarrow 0$ ) so that $\left\|w_{k}\right\|_{L^{2}(\Omega)} \rightarrow 0$. Then (4.15) yields $\gamma\left\|D w_{k}\right\|_{L^{2}(\Omega)} \rightarrow 0$ and consequently $\left\|w_{k}\right\|_{W^{1,2}(\Omega)} \rightarrow 0$, that is a contradiction.

As a byproduct of the above proof we have by (4.12), (4.13), (4.14), for any $u, v \in W^{1,2}(\Omega)$

$\left|\left\langle\mathcal{G}_{\varepsilon}(u), v\right\rangle_{\varepsilon}\right| \leq C\left(\|u\|_{W^{1,2}(\Omega)}\|v\|_{L^{2}(\Omega)}+\|v\|_{W^{1,2}(\Omega)}\left(\|u\|_{L^{2}(\Omega)}+1\right)+\varepsilon\|u\|_{W^{1,2}(\Omega)}\|v\|_{W^{1,2}(\Omega)}\right)$,

where $C$ is independent of $\varepsilon$. In particular,

$$
\left\|\mathcal{G}_{\varepsilon}(u)\right\|_{X_{\varepsilon}^{*}} \leq C\left(\|u\|_{X_{\varepsilon}}+1\right), \forall u \in X_{\varepsilon} .
$$

Then we have, possibly modifying $\kappa_{2}$ in (2.8),

$$
\text { (2.8) holds true for all } u_{\varepsilon} \in X_{\varepsilon} \text {, }
$$

when $\varepsilon \leq \varepsilon_{0}, \lambda \geq \lambda_{0}$.

\section{Limit transition in the surface term and proof of Proposition 7}

$\mathbf{1}$ (Proof of Proposition 17). Let $\Omega^{\prime}$ be a subdomain of $\Omega$ such that $\overline{\Omega^{\prime}} \subset \Omega$, and let us define the linear functional $b_{\varepsilon}$ on $W^{1,2}(\Omega)$ by

$$
b_{\varepsilon} w_{\varepsilon}=\int_{S_{\varepsilon}^{\prime}} q(x, x / \varepsilon)\left(w_{\varepsilon}-\bar{w}_{\varepsilon}\right) \mathrm{d} \sigma .
$$

where $S_{\varepsilon}^{\prime}=\bigcup_{m: Y_{\varepsilon}^{(m)} \cap \Omega^{\prime} \neq \emptyset} S_{\varepsilon} \cap Y_{\varepsilon}^{(m)}$. Clearly, $S_{\varepsilon}^{\prime} \subset S_{\varepsilon}$.

Step 1 (weak convergence of $b_{\varepsilon}$ ). Let us show that

$$
\begin{gathered}
\left\|b_{\varepsilon}\right\| \leq C \text { with } C \text { independent of } \varepsilon, \\
b_{\varepsilon} w \rightarrow \int_{\Omega^{\prime}} \int_{Y \cap S} q(x, y) D w(x) \cdot y \mathrm{~d} \sigma_{y} \mathrm{~d} x \text { weakly, as } \varepsilon \rightarrow 0 .
\end{gathered}
$$


We have by (4.3),

$$
\left|b_{\varepsilon} w_{\varepsilon}\right| \leq C \int_{S_{\varepsilon}^{\prime}}\left|w_{\varepsilon}-\bar{w}_{\varepsilon}\right| \mathrm{d} \sigma \leq C \varepsilon^{-1 / 2}\left(\int_{S_{\varepsilon}^{\prime}}\left|w_{\varepsilon}-\bar{w}_{\varepsilon}\right|^{2} \mathrm{~d} \sigma\right)^{1 / 2} \leq C\left\|w_{\varepsilon}\right\|_{W^{1,2}(\Omega)} .
$$

Now chose an arbitrary $w$ from the dense (in $W^{1,2}(\Omega)$ ) set $C^{2}(\bar{\Omega})$. We have

$$
\begin{aligned}
b_{\varepsilon} w=\sum_{m} \int_{S_{\varepsilon}^{\prime} \cap Y_{\varepsilon}^{(m)}} q(x, x / \varepsilon)\left(D w\left(x_{\varepsilon}^{(m)}\right) \cdot\left(x-x_{\varepsilon}^{(m)}\right)+O\left(\varepsilon^{2}\right)\right) \mathrm{d} \sigma \\
=\sum_{m} \int_{S_{\varepsilon}^{\prime} \cap Y_{\varepsilon}^{(m)}} q\left(x_{\varepsilon}^{(m)}, x / \varepsilon\right) D w\left(x_{\varepsilon}^{(m)}\right) \cdot\left(x-x_{\varepsilon}^{(m)}\right) \mathrm{d} \sigma+O(\varepsilon) \\
=\int_{\Omega^{\prime}} \int_{Y \cap S} q(x, y) D w(x) \cdot y \mathrm{~d} \sigma_{y} \mathrm{~d} x+o(1) .
\end{aligned}
$$

where $x_{\varepsilon}^{(m)}$ is the center of the cell $Y_{\varepsilon}^{(m)}$. Thus (5.2) and (6.23) are proved.

Step 2(Proof of $(3.9)$ for $w_{\varepsilon}$ with $\left.\operatorname{supp}\left(w_{\varepsilon}\right) \subset \overline{\Omega^{\prime}}\right)$. Assume now that

$$
w_{\varepsilon}=0 \text { in } \Omega \backslash \Omega^{\prime} \text { (in particular } w_{\varepsilon}=0 \text { on } \partial \Omega^{\prime} \text { ). }
$$

Given $\delta>0$, let $\left\{Q_{\delta}^{(\alpha)}\right\}$ be an open cover of $\Omega$, $\operatorname{diam} Q_{\delta}^{(\alpha)} \leq \delta$, and let $\left\{\varphi_{\delta}^{(\alpha)} \in\right.$ $\left.C^{\infty}\left(\mathbb{R}^{N}\right)\right\}$ be a partition of unity such that

$$
\operatorname{supp} \varphi_{\delta}^{(\alpha)} \subset Q_{\delta}^{(\alpha)}, 0 \leq \varphi_{\delta}^{(\alpha)} \leq 1, \sum_{\alpha} \varphi_{\delta}^{(\alpha)}=1
$$

Then we have

$$
\begin{aligned}
b_{\varepsilon} w_{\varepsilon}=\sum_{\alpha} \int_{S_{\varepsilon}^{\prime}} & q\left(\hat{x}_{\delta}^{(\alpha)}, x / \varepsilon\right)\left(w_{\varepsilon}-\bar{w}_{\varepsilon}\right) \varphi_{\delta}^{(\alpha)} \mathrm{d} \sigma \\
& \quad+\sum_{\alpha} \int_{S_{\varepsilon}^{\prime}}\left(q(x, x / \varepsilon)-q\left(\hat{x}_{\delta}^{(\alpha)}, x / \varepsilon\right)\right)\left(w_{\varepsilon}-\bar{w}_{\varepsilon}\right) \varphi_{\delta}^{(\alpha)} \mathrm{d} \sigma=I_{1}+I_{2},
\end{aligned}
$$

where $\hat{x}_{\delta}^{(\alpha)} \in Q_{\delta}^{(\alpha)}$. Thanks to the Lipschitz continuity of $q(x, y)$ in $x$,

$$
\left|I_{2}\right| \leq C \delta \sum_{\alpha} \int_{S_{\varepsilon}^{\prime}}\left|w-\bar{w}_{\varepsilon}\right| \varphi_{\delta}^{(\alpha)} \mathrm{d} \sigma=C \delta \int_{S_{\varepsilon}^{\prime}}\left|w-\bar{w}_{\varepsilon}\right| \mathrm{d} \sigma \leq C \delta\left\|D w_{\varepsilon}\right\|_{L^{2}(\Omega)} .
$$

We write the first term $I_{1}$ as

$$
I_{1}=\sum_{\alpha}\left(\int_{S_{\varepsilon}^{\prime}} q\left(\hat{x}_{\delta}^{(\alpha)}, x / \varepsilon\right) w_{\varepsilon} \varphi_{\delta}^{(\alpha)} \mathrm{d} \sigma-\int_{S_{\varepsilon}^{\prime}} q\left(\hat{x}_{\delta}^{(\alpha)}, x / \varepsilon\right) \bar{w}_{\varepsilon} \varphi_{\delta}^{(\alpha)} \mathrm{d} \sigma\right)=\sum_{\alpha}\left(\tilde{I}_{1}^{(\alpha)}+\hat{I}_{1}^{(\alpha)}\right) .
$$


Note that

$$
\int_{S_{\varepsilon}^{\prime} \cap Y_{\varepsilon}^{(m)}} q\left(\hat{x}_{\delta}^{(\alpha)}, x / \varepsilon\right) \varphi_{\delta}^{(\alpha)} \mathrm{d} \sigma=\varepsilon^{N}\left(\int_{S \cap Y} q\left(\hat{x}_{\delta}^{(\alpha)}, y\right) D \varphi_{\delta}^{(\alpha)}\left(x_{\varepsilon}^{(m)}\right) \cdot y \mathrm{~d} \sigma_{y}+O(\varepsilon)\right)
$$

(as above $x_{\varepsilon}^{(m)}$ denotes the center of the cell $Y_{\varepsilon}^{(m)}$ ). Since $\bar{w}_{\varepsilon} \rightarrow W_{0}(x)$ strongly in $L^{2}(\Omega)$, we obtain

$$
\begin{aligned}
\hat{I}_{1}^{(\alpha)} \rightarrow-\int_{\Omega^{\prime}}\left(W_{0}(x) \int_{S \cap Y} q\left(\hat{x}_{\delta}^{(\alpha)}, y\right) D \varphi_{\delta}^{(\alpha)}(x) \cdot y \mathrm{~d} \sigma_{y}\right) \mathrm{d} x \\
\quad=\int_{\Omega^{\prime}}\left(\varphi_{\delta}^{(\alpha)}(x) \int_{S \cap Y} q\left(x_{\delta}^{(\alpha)}, y\right) D W_{0}(x) \cdot y \mathrm{~d} \sigma_{y}\right) \mathrm{d} x
\end{aligned}
$$

where we have used the fact that $W_{0}=0$ in $\Omega \backslash \Omega^{\prime}$. Thus,

$$
\sum_{\alpha} \hat{I}_{1}^{(\alpha)} \rightarrow \int_{S \cap Y}\left(\sum_{\alpha} \int_{\Omega^{\prime}} \varphi_{\delta}^{(\alpha)}(x) q\left(\hat{x}_{\delta}^{(\alpha)}, y\right) D W_{0}(x) \cdot y \mathrm{~d} x\right) \mathrm{d} \sigma_{y}
$$

therefore

$$
\lim _{\delta \rightarrow 0} \lim _{\varepsilon \rightarrow 0} \sum_{\alpha} \hat{I}_{1}^{(\alpha)}=\int_{\Omega^{\prime}} \int_{S \cap Y} q(x, y) D W_{0}(x) \cdot y \mathrm{~d} \sigma_{y} \mathrm{~d} x .
$$

In order to pass to the limit in $\tilde{I}_{1}^{(\alpha)}$ as $\varepsilon \rightarrow 0$, consider the solution $\theta$ of the problem

$$
\left\{\begin{array}{l}
\Delta \theta(y)=0, \text { in } Y^{*} \\
\frac{\partial \theta}{\partial \nu}=q\left(\hat{x}_{\delta}^{(\alpha)}, y\right) \text { on } S \cap Y \\
\theta \text { is } Y^{*}-\text { periodic. }
\end{array}\right.
$$

Thanks to the property (c) of $q(x, y)$ there is a unique (up to an additive constant) solution $\theta$ of (5.9) and $\theta \in W^{1,2}\left(Y^{*}\right)$. Set $\zeta_{\varepsilon}(x)=\theta(x / \varepsilon)$, then we have $\Delta \zeta_{\varepsilon}=0$ in $\Omega_{\varepsilon}$ and $\varepsilon \frac{\partial \zeta_{\varepsilon}}{\partial \nu}=q\left(\hat{x}_{\delta}^{(\alpha)}, x / \varepsilon\right)$ on $S_{\varepsilon}^{\prime}$, so that

$$
\begin{aligned}
\int_{S_{\varepsilon}^{\prime}} q\left(\hat{x}_{\delta}^{(\alpha)}, x / \varepsilon\right) & w_{\varepsilon} \varphi_{\delta}^{(\alpha)} \mathrm{d} \sigma=\varepsilon \int_{S_{\varepsilon}^{\prime}} w_{\varepsilon} \varphi_{\delta}^{(\alpha)} \frac{\partial \zeta_{\varepsilon}}{\partial \nu} \mathrm{d} \sigma \\
= & \varepsilon \int_{\Omega_{\varepsilon} \cap \Omega^{\prime}} D\left(w_{\varepsilon} \varphi_{\delta}^{(\alpha)}\right) \cdot D \zeta_{\varepsilon} \mathrm{d} x=\int_{\Omega_{\varepsilon} \cap \Omega^{\prime}} D\left(w_{\varepsilon} \varphi_{\delta}^{(\alpha)}\right) \cdot(D \theta)(x / \varepsilon) \mathrm{d} x .
\end{aligned}
$$

(we have taken into account here that $w_{\varepsilon}=0$ on $\partial \Omega^{\prime}$ ). One easily checks that $D\left(w_{\varepsilon} \varphi_{\delta}^{(\alpha)}\right)(x) \rightarrow D\left(W_{0} \varphi_{\delta}^{(\alpha)}\right)(x)+\varphi_{\delta}^{(\alpha)} D_{y} W_{1}(x, y)$ two-scale, therefore

$$
\begin{aligned}
\tilde{I}_{1}^{(\alpha)} \rightarrow \int_{\Omega^{\prime}}\left(\int_{Y^{*}}\left(D\left(W_{0} \varphi_{\delta}^{(\alpha)}\right)+\varphi_{\delta}^{(\alpha)} D_{y} W_{1}(x, y)\right) \cdot(D \theta)(y) \mathrm{d} y\right) \mathrm{d} x \\
=\int_{\Omega^{\prime}} \varphi_{\delta}^{(\alpha)}\left(\int_{S \cap Y} W_{1}(x, y) q\left(\hat{x}_{\delta}^{(\alpha)}, y\right) \mathrm{d} \sigma_{y}\right) \mathrm{d} x
\end{aligned}
$$


where we have used (5.9). Thus, taking into account the Lipschitz continuity of $q(x, y)$ in $x$, we get, passing to the limit as $\delta \rightarrow 0$,

$$
\begin{aligned}
\lim _{\delta \rightarrow 0} \lim _{\varepsilon \rightarrow 0} \sum_{\alpha} \tilde{I}_{1}^{(\alpha)}=\sum_{\alpha} \int_{\Omega^{\prime}} \varphi_{\delta}^{(\alpha)}\left(\int_{S \cap Y} W_{1}(x, y) q(x, y) \mathrm{d} \sigma_{y}\right) \mathrm{d} x \\
\quad=\int_{\Omega^{\prime}} \int_{S \cap Y} W_{1}(x, y) q(x, y) \mathrm{d} \sigma_{y} \mathrm{~d} x
\end{aligned}
$$

and we finally obtain by (5.5) - (5.8), (5.10),

$$
\int_{S_{\varepsilon}^{\prime}} q(x, x / \varepsilon)\left(w_{\varepsilon}-\bar{w}_{\varepsilon}\right) \mathrm{d} \sigma \rightarrow \int_{\Omega^{\prime}} \int_{Y \cap S} q(x, y)\left(D W_{0} \cdot y+W_{1}(x, y)\right) \mathrm{d} \sigma_{y} \mathrm{~d} x .
$$

Step 3(general case). Let $\left(w_{\varepsilon}\right)$ be now an arbitrary sequence such that $w_{\varepsilon} \rightarrow W_{0}$ weakly in $W^{1,2}(\Omega)$, and $D w_{\varepsilon} \rightarrow D W_{0}(x)+D_{y} W_{1}(x, y)$ two-scale. Write $w_{\varepsilon}=$ $\left(w_{\varepsilon}-\left(W_{0}+w_{\varepsilon}^{(1)}\right)\right)+w_{\varepsilon}^{(1)}+W_{0}$, where $w_{\varepsilon}^{(1)}$ is the unique solution of the problem

$$
\left\{\begin{array}{l}
\Delta w_{\varepsilon}^{(1)}=0 \text { in } \Omega^{\prime} \\
w_{\varepsilon}^{(1)}=w_{\varepsilon}-W_{0} \text { on } \partial \Omega^{\prime}
\end{array}\right.
$$

extended in $\Omega \backslash \Omega^{\prime}$ by setting $w_{\varepsilon}^{(1)}=w_{\varepsilon}-W_{0}$. Since $w_{\varepsilon}-W_{0} \rightarrow 0$ weakly in $H^{1 / 2}\left(\partial \Omega^{\prime}\right)$, we have

$$
w_{\varepsilon}^{(1)} \rightarrow 0 \text { strongly in } W^{1,2}(K) \text { for any compact } K \subset \Omega^{\prime},
$$

by standard elliptic estimates. This implies, in particular, that $w_{\varepsilon}^{(1)} \rightarrow 0, D w_{\varepsilon}^{(1)} \rightarrow 0$ two-scale. Moreover in view of (4.3), for any compact subset $K$ of $\Omega^{\prime}$,

$$
\begin{array}{r}
\left|b_{\varepsilon} w_{\varepsilon}^{(1)}\right| \leq C \sum_{m: Y_{\varepsilon}^{(m)} \cap K \neq \emptyset} \int_{Y_{\varepsilon}^{(m)}}\left|w_{\varepsilon}^{(1)}-\bar{w}_{\varepsilon}^{(1)}\right| \mathrm{d} \sigma+C \sum_{m: Y_{\varepsilon}^{(m)} \cap K=\emptyset} \int_{Y_{\varepsilon}^{(m)} \cap \Omega^{\prime}}\left|w_{\varepsilon}^{(1)}-\bar{w}_{\varepsilon}^{(1)}\right| \mathrm{d} \sigma \\
\quad \leq C\left(\int_{K_{\delta}}\left|D w_{\varepsilon}^{(1)}\right|^{2} \mathrm{~d} x\right)^{1 / 2}+C\left|\Omega_{\delta}^{\prime} \backslash K\right|^{1 / 2}\left(\int_{\Omega}\left|D w_{\varepsilon}^{(1)}\right|^{2} \mathrm{~d} x\right)^{1 / 2}, \quad \text { (5.13) }
\end{array}
$$

when $\varepsilon \leq \delta / N$, where $C$ is independent of $\varepsilon$ and $\delta, K_{\delta}, \Omega_{\delta}^{\prime}$ are the $\delta$-neighborhoods of $K$ and $\Omega^{\prime}$, respectively, and $\delta>0$ is arbitrary. (The summation in (5.13) is taken over $m$ such that $Y_{\varepsilon}^{(m)} \cap \Omega^{\prime} \neq \emptyset$.) It follows from (5.12), (5.13) that $b_{\varepsilon} w_{\varepsilon}^{(1)} \rightarrow 0$ as $\varepsilon \rightarrow 0$, while, according to the first and second steps,

$$
b_{\varepsilon} W_{0} \rightarrow \int_{\Omega^{\prime}} \int_{Y \cap S} q(x, y) D W_{0} \cdot y \mathrm{~d} \sigma_{y} \mathrm{~d} x,
$$


and

$$
b_{\varepsilon}\left(w_{\varepsilon}-\left(W_{0}+w_{\varepsilon}^{(1)}\right)\right) \rightarrow \int_{\Omega^{\prime}} \int_{Y \cap S} q(x, y) W_{1}(x, y) \mathrm{d} \sigma_{y} \mathrm{~d} x .
$$

Thus (5.11) is proved for any sequence $\left(w_{\varepsilon}\right)$ such that (3.8) holds.

Final step. Set $\Omega^{\prime}=\{x \in \Omega$; $\operatorname{dist}(x, \partial \Omega)>\delta\}$, where $\delta>0$. By using (4.3) we have,

$$
\int_{S_{\varepsilon} \backslash S_{\varepsilon}^{\prime}}\left|w_{\varepsilon}-\bar{w}_{\varepsilon}\right| \mathrm{d} \sigma \leq C \frac{\delta^{1 / 2}}{\varepsilon^{1 / 2}}\left(\int_{S_{\varepsilon} \backslash S_{\varepsilon}^{\prime}}\left|w_{\varepsilon}-\bar{w}_{\varepsilon}\right|^{2} \mathrm{~d} \sigma\right)^{1 / 2} \leq C \delta^{1 / 2}\left\|w_{\varepsilon}\right\|_{W^{1,2}(\Omega)},
$$

for sufficiently small $\varepsilon$, where $C$ is independent of $\delta$ and $\varepsilon$. Therefore (5.14) combined with (5.11) yield (3.9) for any sequence $\left(w_{\varepsilon}\right)$ such that (3.8) holds.

2(Proof of (3.7) $)$. We approximate $U_{0}$ by functions $u_{\delta}^{(1)} \in C^{1}(\bar{\Omega})(\delta>0)$ in the strong topology of $L^{2}(\Omega),\left\|U_{0}-u_{\delta}^{(1)}\right\|_{L^{2}(\Omega)} \leq \delta$. By virtue of Lemma 9, the strong$L^{2}$ convergence of $u_{\varepsilon}$ to $U_{0}$ and Lemma 10 we then have

$$
\begin{array}{r}
\limsup _{\varepsilon \rightarrow 0} \mid \int_{S_{\varepsilon}} g\left(u_{\varepsilon}, x / \varepsilon\right)\left(u_{\varepsilon}-v_{\varepsilon}\right) \mathrm{d} \sigma-\int_{S_{\varepsilon}} g\left(\bar{u}_{\delta}^{(1)}, x / \varepsilon\right)\left(u_{\varepsilon}-v_{\varepsilon}-\bar{u}_{\varepsilon}+\bar{v}_{\varepsilon}\right) \mathrm{d} \sigma \\
-\int_{S_{\varepsilon}} g_{u}^{\prime}\left(\bar{u}_{\delta}^{(1)}, x / \varepsilon\right)\left(\bar{u}_{\delta}^{(1)}-\bar{v}_{\varepsilon}\right)\left(u_{\varepsilon}-\bar{u}_{\varepsilon}\right) \mathrm{d} \sigma \mid \leq C \delta .
\end{array}
$$

On the other hand, the regularity of $g(u, y)$ in $u$ (conditions (2.3), (2.4), (2.5)) implies the pointwise bounds

$$
\begin{gathered}
\left|g\left(\bar{u}_{\delta}^{(1)}, x / \varepsilon\right)-g\left(u_{\delta}^{(1)}, x / \varepsilon\right)\right| \leq C \varepsilon \text { on } S_{\varepsilon}, \\
\left|g_{u}^{\prime}\left(\bar{u}_{\delta}^{(1)}, x / \varepsilon\right)\left(\bar{u}_{\delta}^{(1)}-\bar{v}_{\varepsilon}\right)-g_{u}^{\prime}\left(u_{\delta}^{(1)}, x / \varepsilon\right)\left(u_{\delta}^{(1)}-V_{0}\right)\right| \leq C \varepsilon \text { on } S_{\varepsilon}
\end{gathered}
$$

(recall that $v_{\varepsilon}=V_{0}(x)+\varepsilon V_{1}(x, x / \varepsilon)$, and $V_{0}, V_{1}$ are smooth functions), which, by using (4.3), lead to

$$
\begin{aligned}
& \limsup _{\varepsilon \rightarrow 0} \mid \int_{S_{\varepsilon}}\left(g\left(\bar{u}_{\delta}^{(1)}, x / \varepsilon\right)-g\left(u_{\delta}^{(1)}, x / \varepsilon\right)\right)\left(u_{\varepsilon}-v_{\varepsilon}-\bar{u}_{\varepsilon}+\bar{v}_{\varepsilon}\right) \mathrm{d} \sigma \\
& \quad+\int_{S_{\varepsilon}}\left(g_{u}^{\prime}\left(\bar{u}_{\delta}^{(1)}, x / \varepsilon\right)\left(\bar{u}_{\delta}^{(1)}-\bar{v}_{\varepsilon}\right)-g_{u}^{\prime}\left(u_{\delta}^{(1)}, x / \varepsilon\right)\left(u_{\delta}^{(1)}-V_{0}\right)\right)\left(u_{\varepsilon}-\bar{u}_{\varepsilon}\right) \mathrm{d} \sigma \mid=0 .
\end{aligned}
$$

Now, applying Proposition 7 first with $q(x, y)=g\left(u_{\delta}^{(1)}(x), y\right), w_{\varepsilon}=u_{\varepsilon}-v_{\varepsilon}$, then with $q(x, y)=g\left(u_{\delta}^{(1)}(x), y\right) u_{\delta}^{(1)}(x), w_{\varepsilon}=u_{\varepsilon}$, and finally with $q(x, y)=g\left(u_{\delta}^{(1)}(x), y\right) V_{0}(x)$, 


$$
\begin{aligned}
& w_{\varepsilon}=u_{\varepsilon} \text {, we get } \\
& \qquad \begin{array}{r}
\int_{S_{\varepsilon}}\left(g\left(u_{\delta}^{(1)}, x / \varepsilon\right)\left(u_{\varepsilon}-v_{\varepsilon}-\bar{u}_{\varepsilon}+\bar{v}_{\varepsilon}\right)+g_{u}^{\prime}\left(u_{\delta}^{(1)}, x / \varepsilon\right)\left(u_{\delta}^{(1)}-V_{0}\right)\left(u_{\varepsilon}-\bar{u}_{\varepsilon}\right)\right) \mathrm{d} \sigma \\
\quad \rightarrow \int_{\Omega} \int_{S \cap Y} g\left(u_{\delta}^{(1)}, y\right)\left(D\left(U_{0}-V_{0}\right) \cdot y+U_{1}(x, y)-V_{1}(x, y)\right) \mathrm{d} \sigma_{y} \mathrm{~d} x \\
\quad+\int_{\Omega} \int_{S \cap Y} g_{u}^{\prime}\left(u_{\delta}^{(1)}, y\right)\left(u_{\delta}^{(1)}-V_{0}\right)\left(D U_{0} \cdot y+U_{1}(x, y)\right) \mathrm{d} \sigma_{y} \mathrm{~d} x .
\end{array}
\end{aligned}
$$

Assuming $\delta \rightarrow 0$ in (5.15), (5.16), (5.17) yields (3.7).

\section{Homogenization of the parabolic problem (1.2)}

In terms of the operators $\mathcal{A}_{\varepsilon}$ and $\mathcal{G}_{\varepsilon}$ problem (1.2) is as follows

$$
\left\{\begin{array}{l}
\partial_{t} u_{\varepsilon}(t)+\mathcal{A}_{\varepsilon}\left(u_{\varepsilon}(t)\right)-\mathcal{G}_{\varepsilon}\left(u_{\varepsilon}(t)\right)=f(t), t>0 \\
u_{\varepsilon}(0)=\tilde{u}
\end{array}\right.
$$

We study the asymptotic behavior of solutions $u^{\varepsilon}$ of (6.1) as $\varepsilon \rightarrow 0$ adapting the notion of two-scale convergence to functions depending on the time variable $t$ which is treated as a parameter. Namely, following [6] we say that

the sequence $v_{\varepsilon}=v_{\varepsilon}(x, t)$ which is bounded in $L^{2}(\Omega \times[0, T])$

two-scale converges to $V_{0}(x, y, t)$ if

$$
\int_{0}^{T} \int_{\Omega} v_{\varepsilon} \phi(x, x / \varepsilon, t) \mathrm{d} x \mathrm{~d} t \rightarrow \int_{0}^{T} \int_{Y} \int_{\Omega} V_{0} \phi(x, y, t) \mathrm{d} x \mathrm{~d} y \mathrm{~d} t
$$

for any $Y-$ periodic in $y$ function $\phi(x, y, t) \in C^{\infty}(\Omega \times Y \times[0, T])$.

The basic properties of the convergence (6.2) are similar to that of the standard twoscale convergence. Namely, any bounded in $L^{2}(\Omega \times[0, T])$ sequence has a subsequence converging in the sense of ([6.2) ; if $\left\|v_{\varepsilon}\right\|_{L^{2}\left(0, T ; W^{1,2}(\Omega)\right)} \leq C$ then, up to extracting a subsequence, $v_{\varepsilon}$ and $D v_{\varepsilon}$ converge in the sense of (6.2) to $V_{0}$ and $D V_{0}(x, t)+D_{y} V_{1}(x, y, t)$ correspondingly, where $V_{0} \in L^{2}\left(0, T ; W^{1,2}(\Omega)\right), V_{1} \in L^{2}\left([0, T] \times \Omega ; W_{\text {per }}^{1,2}(Y)\right)$. Note, however, that (6.2) does not imply, in general, that $v_{\varepsilon}(\cdot, t)$ converges in two-scale sense for a.e. $t \in[0, T]$, but rather

$$
\int_{\alpha}^{\beta} v_{\varepsilon} \mathrm{d} t \rightarrow \int_{\alpha}^{\beta} V_{0} \mathrm{~d} t \text { two scale for all } 0 \leq \alpha<\beta \leq T .
$$


1 (Well-posedness of problem (6.1)). Given $T>0$, let us show that problem (6.1) has a unique solution on the time interval $[0, T]$. To this end we first note that the operator $\mathcal{A}_{\varepsilon}(u)-\mathcal{G}_{\varepsilon}(u)+\tilde{\lambda} u$ becomes monotone if one chooses a suitable $\tilde{\lambda}>0$ (depending on $\varepsilon$ ). Indeed, by using (2.4) we get

$$
\begin{aligned}
\left\langle\mathcal{G}_{\varepsilon}(u)-\mathcal{G}_{\varepsilon}(v),\right. & u-v\rangle_{\varepsilon} \leq C \int_{S_{\varepsilon}}|u-v|^{2} \mathrm{~d} \sigma \\
& \leq \kappa / 2\|D(u-v)\|_{L^{2}\left(\Omega_{\varepsilon}\right)}^{2}+\Gamma_{\varepsilon}\|u-v\|_{L^{2}\left(\Omega_{\varepsilon}\right)}^{2}, \forall u, v \in W^{1,2}\left(\Omega_{\varepsilon}\right) .
\end{aligned}
$$

where $\kappa$ is the constant appearing in (2.1), and $\Gamma_{\varepsilon}$ is independent of $u_{\varepsilon}$ and $v_{\varepsilon}$ (the last inequality in ([6.3) is due the compactness of the trace operator $T_{\varepsilon}: W^{1,2}\left(\Omega_{\varepsilon}\right) \rightarrow$ $L^{2}\left(S_{\varepsilon}\right), T_{\varepsilon} w=$ trace of $w$ on $\left.S_{\varepsilon}\right)$. Then, setting $\tilde{\lambda}=\Gamma_{\varepsilon}+1$, by (2.1) and (6.3) one easily verifies that

$$
\text { the operator } u \mapsto \mathcal{A}_{\varepsilon}(u)-\mathcal{G}_{\varepsilon}(u)+\tilde{\lambda} u \text { is monotone }
$$

By changing the unknown $v_{\varepsilon}=e^{-\tilde{\lambda} t} u_{\varepsilon}$ problem (6.1) is reduced to the evolution problem for the equation $\partial_{t} v_{\varepsilon}(t)+\tilde{\mathcal{A}}_{\varepsilon}\left(v_{\varepsilon}(t), t\right)-\tilde{\mathcal{G}}_{\varepsilon}\left(v_{\varepsilon}(t), t\right)+\tilde{\lambda} v_{\varepsilon}=e^{-\tilde{\lambda} t} f(t), t>0$ with the initial condition $v_{\varepsilon}(0)=\tilde{u}$, where $\tilde{\mathcal{A}}_{\varepsilon}: v \mapsto e^{-\tilde{\lambda} t} \mathcal{A}_{\varepsilon}\left(e^{\tilde{\lambda} t} v\right)$ and $\tilde{\mathcal{G}}_{\varepsilon}: v \mapsto$ $e^{-\tilde{\lambda} t} \mathcal{G}_{\varepsilon}\left(e^{\tilde{\lambda} t} v\right)$. By the standard theory of parabolic problems for monotone operators (see, e.g. [21]) it follows from (6.4), (2.1) and (6.3) that the latter problem has a unique solution on $[0, T]$ as far as $f \in L^{2}\left([0, T] ; X_{\varepsilon}^{*}\right)$ and $\tilde{u} \in L^{2}(\Omega)$.

2 (Uniform a-priori bounds). Let us show that for any $T>0$ the solution $u_{\varepsilon}$ of (6.1) satisfies the following bounds for sufficiently small $\varepsilon$,

$$
\left\|\partial_{t} u_{\varepsilon}\right\|_{L^{2}\left(0, T ; X_{\varepsilon}^{*}\right)}^{2},\left\|u_{\varepsilon}\right\|_{L^{2}\left(0, T ; X_{\varepsilon}\right)}^{2} \leq C\left(\langle\tilde{u}, \tilde{u}\rangle_{\varepsilon}+\|f\|_{L^{2}\left(0, T ; X_{\varepsilon}^{*}\right)}^{2}+1\right),
$$

with a constant $C$ independent of $\varepsilon$. Let $\varepsilon_{0}, \lambda_{0}$ be as in Theorem 11. From (6.1) we have, for $\varepsilon \leq \varepsilon_{0}$

$$
\begin{aligned}
\left\langle u_{\varepsilon}(t), u_{\varepsilon}(t)\right\rangle_{\varepsilon}+2 \int_{0}^{t}\left\langle\mathcal{A}_{\varepsilon}\left(u_{\varepsilon}(\tau)\right)\right. & \left.+\mathcal{G}_{\varepsilon}\left(u_{\varepsilon}(\tau)\right)+\lambda_{0} u_{\varepsilon}(\tau), u_{\varepsilon}(\tau)\right\rangle_{\varepsilon} \mathrm{d} \tau \\
& =\langle\tilde{u}, \tilde{u}\rangle_{\varepsilon}+2 \int_{0}^{t}\left\langle f(\tau)+\lambda_{0} u_{\varepsilon}(\tau), u_{\varepsilon}(\tau)\right\rangle_{\varepsilon} \mathrm{d} \tau
\end{aligned}
$$


Then (6.6) combined with (4.18) yields

$$
\begin{array}{r}
\left\langle u_{\varepsilon}\left(T^{\prime}\right), u_{\varepsilon}\left(T^{\prime}\right)\right\rangle_{\varepsilon}+2 \kappa_{1}\left\|u_{\varepsilon}\right\|_{L^{2}\left(0, T^{\prime} ; X_{\varepsilon}\right)}^{2} \leq\langle\tilde{u}, \tilde{u}\rangle_{\varepsilon}+\|f\|_{L^{2}\left(0, T^{\prime} ; X_{\varepsilon}^{*}\right)}\left\|u_{\varepsilon}\right\|_{L^{2}\left(0, T^{\prime} ; X_{\varepsilon}\right)} \\
+2 T^{\prime} \kappa_{2}+2 \lambda_{0} \int_{0}^{T^{\prime}}\left\langle u_{\varepsilon}(t), u_{\varepsilon}(t)\right\rangle_{\varepsilon} \mathrm{d} t, \quad \forall 0 \leq T^{\prime} \leq T .
\end{array}
$$

Therefore

$$
\left\langle u_{\varepsilon}\left(T^{\prime}\right), u_{\varepsilon}\left(T^{\prime}\right)\right\rangle_{\varepsilon} \leq e^{2 \lambda_{0} T^{\prime}}\left(\langle\tilde{u}, \tilde{u}\rangle_{\varepsilon}+\frac{1}{\kappa_{1}}\|f\|_{L^{2}\left(0, T^{\prime} ; X_{\varepsilon}^{*}\right)}^{2}+2 T^{\prime} \kappa_{2}\right),
$$

combined with (6.7) this implies the second bound in (6.5); while $\left\|\partial_{t} u_{\varepsilon}\right\|_{L^{2}\left(0, T ; X_{\varepsilon}^{*}\right)} \leq$ $\left\|\mathcal{A}_{\varepsilon}\left(u_{\varepsilon}\right)\right\|_{L^{2}\left(0, T ; X_{\varepsilon}^{*}\right)}+\left\|\mathcal{G}_{\varepsilon}\left(u_{\varepsilon}\right)\right\|_{L^{2}\left(0, T ; X_{\varepsilon}^{*}\right)}+\|f\|_{L^{2}\left(0, T ; X_{\varepsilon}^{*}\right)}$ and thus the first bound in (6.5) is a consequence of the second one and (4.17).

3 (Homogenization of problem (6.1)). Let $u_{\varepsilon}$ be continued in $x$ variable onto $\Omega$ by using the extension operator $P_{\varepsilon}$, then the resulting function, still denoted $u_{\varepsilon}$, satisfies

$$
\left\|u_{\varepsilon}(t)\right\|_{L^{2}(\Omega)} \leq C \text { for all } t \in[0, T] \text {, and }\left\|u_{\varepsilon}\right\|_{L^{2}\left(0, T ; W^{1,2}(\Omega)\right)} \leq C,
$$

with a constant $C$ independent of $\varepsilon$. This implies that, up to extracting a subsequence,

$$
\begin{gathered}
\left.u_{\varepsilon} \rightarrow U_{0}(x, t) \text { two-scale (in the sense of }(\underline{6.2})\right) \text { and weakly in } L^{2}\left(0, T ; W^{1,2}(\Omega)\right. \text { ), } \\
\left.D_{x} u_{\varepsilon} \rightarrow D_{x} U_{0}(x, t)+D_{y} U_{1}(x, y, t) \text { two-scale (in the sense of (6.10) }\right) \text {, }
\end{gathered}
$$

where $U_{0} \in L^{2}\left(0, T ; W^{1,2}(\Omega)\right), U_{1} \in L^{2}\left(0, T ; L^{2}\left(\Omega ; W_{p e r}^{1,2}(Y)\right)\right)$. Besides, if we set $\hat{u}_{\varepsilon}=u_{\varepsilon}$ when $x \in \Omega_{\varepsilon}$ and $\hat{u}_{\varepsilon}=0$ when $x \in \Omega \backslash \Omega_{\varepsilon}$, then (6.10) yields that $\hat{u}_{\varepsilon} \rightarrow\left|Y^{*}\right| U_{0}(x, t)$ weakly in $L^{2}\left(0, T ; L^{2}(\Omega)\right)$.

Let $X=W^{1,2}(\Omega)$ an let $X^{*}$ be its dual with respect to the duality pairing

$$
\langle u, v\rangle=\left|Y^{*}\right| \int_{\Omega} u v \mathrm{~d} x .
$$

Show that $U_{0} \in W^{1,2}\left(0, T ; X^{*}\right)$, and $\hat{u}_{\varepsilon}(t) \rightarrow\left|Y^{*}\right| U_{0}(t)$ weakly in $L^{2}(\Omega)$ for all $0 \leq t \leq T$. From (6.10) we have, for any $\phi \in X$ and $\varphi \in C_{0}^{\infty}([0, T])$,

$$
\int_{0}^{T}\left\langle\partial_{t} u_{\varepsilon}, \phi\right\rangle_{\varepsilon} \varphi(t) \mathrm{d} t=-\int_{0}^{T}\left\langle u_{\varepsilon}, \phi\right\rangle_{\varepsilon} \varphi^{\prime}(t) \mathrm{d} t \rightarrow-\int_{0}^{T}\left\langle U_{0}, \phi\right\rangle \varphi^{\prime}(t) \mathrm{d} t .
$$


On the other hand, by using (ㅎ․5 $)$, we get

$$
\left|\int_{0}^{T}\left\langle\partial_{t} u_{\varepsilon}, \phi\right\rangle_{\varepsilon} \varphi(t) \mathrm{d} t\right|^{2} \leq C \int_{0}^{T}\|\phi\|_{X_{\varepsilon}}^{2}|\varphi(t)|^{2} \mathrm{~d} t \leq C\|\varphi \phi\|_{L^{2}(0, T ; X)}^{2} .
$$

Then (6.12), (6.13) show that $U_{0} \in W^{1,2}\left(0, T ; X^{*}\right)$. According to (6.8), the norms $\left\|\hat{u}_{\varepsilon}(t)\right\|_{L^{2}(\Omega)}$ are uniformly in $0<\varepsilon \leq \varepsilon_{0}$ and $t \in[0, T]$ bounded. Thus, to prove that $\hat{u}_{\varepsilon}(t) \rightarrow\left|Y^{*}\right| U_{0}(t)$ weakly in $L^{2}(\Omega)$ for every $t \in[0, T]$ it suffices to show that

$$
\left\langle u_{\varepsilon}(t), \phi\right\rangle_{\varepsilon} \rightarrow\left\langle U_{0}(t), \phi\right\rangle \text { for any } \phi \in X
$$

By the first bound in (6.5) we have $\left|\left\langle u_{\varepsilon}(t)-u_{\varepsilon}\left(t^{\prime}\right), \phi\right\rangle_{\varepsilon}\right| \leq C\left|t-t^{\prime}\right|^{1 / 2}\|\phi\|_{X}$, on the other hand (6.14) holds in the sense of weak star convergence in $L^{\infty}(0, T)$ since $\hat{u}_{\varepsilon} \rightarrow\left|Y^{*}\right| U_{0}(x, t)$ weakly in $L^{2}\left(0, T ; L^{2}(\Omega)\right)$. Thus (6.14) holds for any $t \in[0, T]$, so that $\forall t \in[0, T] \hat{u}_{\varepsilon}(t) \rightarrow\left|Y^{*}\right| U_{0}(t)$ weakly in $L^{2}(\Omega)$, in particular,

$$
\begin{array}{r}
\liminf _{\varepsilon \rightarrow 0}\left\langle u_{\varepsilon}(T), u_{\varepsilon}(T)\right\rangle_{\varepsilon}=\liminf _{\varepsilon \rightarrow 0} \int_{\Omega_{\varepsilon}}\left(\left(u_{\varepsilon}(T)-U_{0}(T)\right)^{2}-U_{0}^{2}(T)\right) \mathrm{d} x \\
+2 \lim _{\varepsilon \rightarrow 0} \int_{\Omega} \hat{u}_{\varepsilon}(T) U_{0}(T) \mathrm{d} x=\liminf _{\varepsilon \rightarrow 0} \int_{\Omega_{\varepsilon}}\left(u_{\varepsilon}(T)-U_{0}(T)\right)^{2} \mathrm{~d} x+\left\langle U_{0}(T), U_{0}(T)\right\rangle \\
\geq\left\langle U_{0}(T), U_{0}(T)\right\rangle,
\end{array}
$$

and, clearly,

$$
\left\langle u_{\varepsilon}(T), v_{\varepsilon}\right\rangle_{\varepsilon} \rightarrow\left\langle U_{0}(T), V_{0}\right\rangle \text {, for any sequence } v_{\varepsilon} \rightarrow V_{0} \text { strongly in } L^{2}(\Omega) \text {. }
$$

Lemma 11. If $\left(u_{\varepsilon}\right)$ is such a (sub) sequence of solutions of (6.1) that (6.10) holds, then

$$
\left\|u_{\varepsilon}-U_{0}\right\|_{L^{2}(\Omega \times[0, T])} \rightarrow 0 \text { as } \varepsilon \rightarrow 0 .
$$

Proof. By (6.10) it suffices to establish the (relative) compactness of $\left(u_{\varepsilon}\right)$ in $L^{2}(\Omega \times$ $[0, T])$. This is achieved by constructing a sequence of compacts $K_{k}(k=1,2, \ldots)$ in $L^{2}(\Omega \times[0, T])$ such that $\lim _{k \rightarrow \infty} \limsup _{\varepsilon \rightarrow 0} \operatorname{dist}_{L^{2}(\Omega \times[0, T])}\left(u_{\varepsilon}, K_{k}\right)=0$.

Let $0=\omega_{\varepsilon}^{(0)}<\omega_{\varepsilon}^{(1)} \leq \ldots \leq \omega_{\varepsilon}^{(j)} \leq \ldots$ be the spectrum of the Neumann eigenvalue problem

$$
\left\{\begin{array}{l}
-\Delta \phi=\omega \phi \text { in } \Omega_{\varepsilon} \\
\frac{\partial \phi}{\partial \nu}=0 \text { on } \partial \Omega_{\varepsilon}
\end{array}\right.
$$


The eigenfunctions $\phi_{\varepsilon}^{(j)}$ can be chosen to form an orthogonal basis of $L^{2}\left(\Omega_{\varepsilon}\right)$, then

$$
u_{\varepsilon}(t)=\sum_{j=0}^{\infty} f_{\varepsilon}^{(j)}(t) P_{\varepsilon} \phi_{\varepsilon}^{(j)}, \text { where } f_{\varepsilon}^{(j)}(t)=\left\langle u_{\varepsilon}(t), \phi_{\varepsilon}^{(j)}\right\rangle_{\varepsilon}
$$

Moreover $\phi_{\varepsilon}^{(j)} /\left(\omega_{\varepsilon}^{(j)}+1\right)^{1 / 2}$ form an orthonormal basis in $X_{\varepsilon}\left(=W^{1,2}\left(\Omega_{\varepsilon}\right)\right.$, hence

$$
\sum_{j=0}^{\infty}\left(1+\omega_{\varepsilon}^{(j)}\right) \int_{0}^{T}\left|f_{\varepsilon}^{(j)}(t)\right|^{2} \mathrm{~d} t=\left\|u_{\varepsilon}\right\|_{L^{2}\left(0, T ; X_{\varepsilon}\right)}^{2} \leq\left\|u_{\varepsilon}\right\|_{L^{2}(0, T ; X)}^{2} \leq C .
$$

It is well known that $\omega_{\varepsilon}^{(k)} \rightarrow \omega^{(k)}$ as $\varepsilon \rightarrow 0$, where $0=\omega^{(0)}<\omega^{(1)} \leq \cdots \leq \omega^{(j)} \leq$ ... is the discrete spectrum of a homogenized problem. By the first bound in (6.5) we have $\left|f_{\varepsilon}^{(j)}(t)-f_{\varepsilon}^{(j)}\left(t^{\prime}\right)\right| \leq C\left|t-t^{\prime}\right|^{1 / 2}\left\|\phi_{\varepsilon}^{(j)}\right\|_{X_{\varepsilon}}=C\left|t-t^{\prime}\right|^{1 / 2}\left(1+\omega_{\varepsilon}^{(j)}\right)^{1 / 2}$ for all $t, t^{\prime} \in$ $[0, T]$. It follows that, for every $k$ fixed, the sequence $\left(u_{\varepsilon}^{(k)}:=\sum_{j=0}^{k} f_{\varepsilon}^{(j)}(t) P_{\varepsilon} \phi_{\varepsilon}^{(j)}\right)$ is in a bounded closed subset $K_{k}$ of $C^{1 / 2}([0, T] ; X)$. Clearly $K_{k}$ is a compact set in $L^{2}(\Omega \times[0, T])$. On the other hand, due to the properties of the extension operator $P_{\varepsilon}$

$$
\left\|u_{\varepsilon}-u_{\varepsilon}^{(k)}\right\|_{L^{2}(\Omega \times[0, T])}^{2} \leq C \int_{0}^{T}\left\|u_{\varepsilon}-u_{\varepsilon}^{(k)}\right\|_{L^{2}\left(\Omega_{\varepsilon}\right)}^{2} \mathrm{~d} t=C \sum_{j=k+1}^{\infty} \int_{0}^{T}\left|f_{\varepsilon}^{(j)}(t)\right|^{2} \mathrm{~d} t
$$

therefore, in view of (6.18), $\limsup _{\varepsilon \rightarrow 0} \operatorname{dist}_{L^{2}(\Omega \times[0, T])}\left(u_{\varepsilon}, K_{k}\right) \leq \limsup _{\varepsilon \rightarrow 0} \| u_{\varepsilon}-$ $u_{\varepsilon}^{(k)} \|_{L^{2}(\Omega \times[0, T])} \leq C / \omega^{(k+1)} \rightarrow 0$ as $k \rightarrow \infty$.

Now, set $V_{0}(x, t) \in C^{\infty}(\bar{\Omega} \times[0, T]), V_{1}(x, y, t) \in C^{\infty}(\bar{\Omega} \times \bar{Y} \times[0, T])$ with $V_{1}(x, y, t)$ being $Y$-periodic in $y$, set $v_{\varepsilon}=V_{0}(x, t)+\varepsilon V_{1}(x, x / \varepsilon, t)$, and using the test function $w_{\varepsilon}=u_{\varepsilon}-v_{\varepsilon}$ in (6.1) we obtain

$$
\begin{aligned}
\frac{1}{2}\left\langle u_{\varepsilon}(T), u_{\varepsilon}(T)\right\rangle_{\varepsilon}-\frac{1}{2}\langle\tilde{u}, \tilde{u}\rangle_{\varepsilon}-\left\langle u_{\varepsilon}(T), v_{\varepsilon}(T)\right\rangle_{\varepsilon}+\left\langle\tilde{u}, v_{\varepsilon}(0)\right\rangle_{\varepsilon} & \\
+\int_{0}^{T}\left\langle u_{\varepsilon}(t), \partial_{t} v_{\varepsilon}(t)\right\rangle_{\varepsilon} \mathrm{d} t+\int_{0}^{T}\left\langle\mathcal{A}_{\varepsilon}\left(u_{\varepsilon}(t)\right), w_{\varepsilon}(t)\right\rangle_{\varepsilon} \mathrm{d} t & -\int_{0}^{T}\left\langle\mathcal{G}_{\varepsilon}\left(u_{\varepsilon}(t)\right), w_{\varepsilon}(t)\right\rangle_{\varepsilon} \mathrm{d} t \\
& =\int_{0}^{T}\left\langle f(t), w_{\varepsilon}(t)\right\rangle_{\varepsilon} \mathrm{d} t .
\end{aligned}
$$

By using (6.10) and (6.15), (6.16), we can take $\lim ^{6.6 n f}{ }_{\varepsilon \rightarrow 0}$ for various terms in (6.19) 
to get

$$
\begin{aligned}
\liminf _{\varepsilon \rightarrow 0}\left(\frac{1}{2}\left\langle u_{\varepsilon}(T), u_{\varepsilon}(T)\right\rangle_{\varepsilon}\right. & -\frac{1}{2}\langle\tilde{u}, \tilde{u}\rangle_{\varepsilon}-\left\langle u_{\varepsilon}(T), v_{\varepsilon}(T)\right\rangle_{\varepsilon}+\left\langle\tilde{u}, v_{\varepsilon}(0)\right\rangle_{\varepsilon} \\
& \left.+\int_{0}^{T}\left(\left\langle u_{\varepsilon}(t), \partial_{t} v_{\varepsilon}(t)\right\rangle_{\varepsilon}-\left\langle f(t), w_{\varepsilon}(t)\right\rangle_{\varepsilon}\right) \mathrm{d} t\right) \\
\geq \frac{1}{2}\left\langle U_{0}(T), U_{0}(T)\right\rangle-\frac{1}{2}\langle\tilde{u}, \tilde{u}\rangle-\left\langle U_{0}(T), V_{0}(T)\right\rangle+\left\langle\tilde{u}, V_{0}(0)\right\rangle & \\
& +\int_{0}^{T}\left(\left\langle U_{0}(t), \partial_{t} V_{0}(t)\right\rangle-\left\langle f(t), U_{0}(t)-V_{0}(t)\right\rangle\right) \mathrm{d} t
\end{aligned}
$$

By (6.11) we also have

$$
\begin{aligned}
& \lim _{\varepsilon \rightarrow 0} \int_{0}^{T}\left\langle\mathcal{A}_{\varepsilon}\left(v_{\varepsilon}(t)\right), w_{\varepsilon}(t)\right\rangle_{\varepsilon} \mathrm{d} t \\
& =\int_{0}^{T} \int_{\Omega} \int_{Y^{*}} a\left(D_{x} V_{0}+D_{y} V_{1}, y\right) \cdot\left(D_{x} U_{0}+D_{y} U_{1}-D_{x} V_{0}-D_{y} V_{1}\right) \mathrm{d} y \mathrm{~d} x \mathrm{~d} t .
\end{aligned}
$$

Let us show that

$$
\int_{0}^{T}\left\langle\mathcal{G}_{\varepsilon}\left(u_{\varepsilon}\right), u_{\varepsilon}-v_{\varepsilon}\right\rangle_{\varepsilon} \mathrm{d} t \rightarrow \int_{0}^{T} M\left(U_{0}, U_{1}, V_{0}, V_{1}\right) \mathrm{d} t \text { as } \varepsilon \rightarrow 0
$$

where $M\left(U_{0}, U_{1}, V_{0}, V_{1}\right)$ is given by (3.12) (or, equivalently, by the r.h.s. of (3.7)) . The proof of (6.22) follows closely the arguments in the end of Sec. 5 (proof of (3.7)). In place of Proposition 7 we make use now of

Proposition 12. Assume that $q(t, x, y) \in C\left([0, T] \times \Omega ; L^{\infty}(S)\right)$ satisfies, (a) $\left|q(t, x, y)-q\left(t^{\prime}, x^{\prime}, y\right)\right| \leq C\left(\left|x-x^{\prime}\right|+\left|t-t^{\prime}\right|\right)$ with $C>0$ independent of $x, x^{\prime} \in \Omega$, $t, t^{\prime} \in[0, T]$ and $y \in S$; (b) $q(t, x, y)$ is $Y$-periodic in $y \in S$;

(c) $\int_{Y \cap S} q(t, x, y) \mathrm{d} \sigma_{y}=0$ for all $x \in \Omega, t \in[0, T]$.

Then, given a sequence $w_{\varepsilon} \in L^{2}\left(0, T ; W^{1,2}(\Omega)\right)$ such that $w_{\varepsilon} \rightarrow W_{0}, D_{x} w_{\varepsilon}(x, t) \rightarrow$ $D_{x} W_{0}(x, t)+D_{y} W_{1}(x, y, t)$ two scale (in the sense of ([6.2) ) as $\varepsilon \rightarrow 0$, we have

$$
\int_{0}^{T} \int_{S_{\varepsilon}} q(t, x, x / \varepsilon)\left(w_{\varepsilon}-\bar{w}_{\varepsilon}\right) \mathrm{d} \sigma \mathrm{d} t \rightarrow \int_{0}^{T} \int_{\Omega} \int_{Y \cap S} q(t, x, y)\left(D_{x} W_{0} \cdot y+W_{1}\right) \mathrm{d} \sigma_{y} \mathrm{~d} x \mathrm{~d} t .
$$

Proof. Set $0=t_{0}^{(n)}<\cdots<t_{j}^{(n)}=T j / n<\cdots<t_{n}^{(n)=T}, \Delta_{j}^{(n)}=\left(t_{j-1}^{(n)}, t_{j}^{(n)}\right)$, then by 
using (4.3) and the Lipschitz continuity of $q(t, x, y)$ in $t$ we obtain

$$
\begin{array}{r}
\int_{0}^{T} \int_{S_{\varepsilon}} q(t, x, x / \varepsilon)\left(w_{\varepsilon}-\bar{w}_{\varepsilon}\right) \mathrm{d} \sigma \mathrm{d} t=\sum_{j=1}^{n} \int_{\Delta_{j}^{(n)}} \int_{S_{\varepsilon}} q(t, x, x / \varepsilon)\left(w_{\varepsilon}-\bar{w}_{\varepsilon}\right) \mathrm{d} \sigma \mathrm{d} t \\
=\sum_{j=1}^{n} \int_{S_{\varepsilon}} q\left(t_{j}^{(n)}, x, x / \varepsilon\right) \int_{\Delta_{j}^{(n)}}\left(w_{\varepsilon}-\bar{w}_{\varepsilon}\right) \mathrm{d} t \mathrm{~d} \sigma+r_{\varepsilon}^{(n)}
\end{array}
$$

with

$$
\left|r_{\varepsilon}^{(n)}\right| \leq \frac{C}{n} \int_{0}^{T} \int_{S_{\varepsilon}}\left|w_{\varepsilon}-\bar{w}_{\varepsilon}\right| \mathrm{d} \sigma \mathrm{d} t \leq \frac{C}{n} \int_{0}^{T}\left\|w_{\varepsilon}\right\|_{W^{1,2}(\Omega)} \mathrm{d} t .
$$

Setting $W_{\varepsilon}=\int_{\Delta_{j}^{(n)}} w_{\varepsilon} \mathrm{d} t$ and applying Proposition [7, we get

$$
\lim _{\varepsilon \rightarrow 0} \int_{S_{\varepsilon}} q\left(t_{j}^{(n)}, x, x / \varepsilon\right)\left(W_{\varepsilon}-\bar{W}_{\varepsilon}\right) \mathrm{d} t \mathrm{~d} \sigma=\int_{\Delta_{j}^{(n)}} \int_{\Omega} \int_{Y \cap S} q\left(t_{j}^{(n)}, x, y\right)\left(D_{x} W_{0} \cdot y+W_{1}\right) \mathrm{d} \sigma_{y} \mathrm{~d} x \mathrm{~d} t .
$$

If we pass to the limit (along a subsequence) as $\varepsilon \rightarrow 0$ in (6.24) and send $n$ to $\infty$ in the resulting relation, then by (6.25) and (6.26) we obtain (6.23).

Proof of (6.22) (continued). By virtue of Lemma 9 and (6.9) we have

$$
\begin{aligned}
\int_{0}^{T}\left\langle\mathcal{G}_{\varepsilon}\left(u_{\varepsilon}\right), u_{\varepsilon}-v_{\varepsilon}\right\rangle_{\varepsilon} \mathrm{d} t & =\int_{0}^{T} \int_{S_{\varepsilon}} g\left(\bar{u}_{\varepsilon}, x / \varepsilon\right)\left(u_{\varepsilon}-v_{\varepsilon}-\bar{u}_{\varepsilon}+\bar{v}_{\varepsilon}\right) \mathrm{d} \sigma \mathrm{d} t \\
& +\int_{0}^{T} \int_{S_{\varepsilon}} g_{u}^{\prime}\left(\bar{u}_{\varepsilon}, x / \varepsilon\right)\left(\bar{u}_{\varepsilon}-\bar{v}_{\varepsilon}\right)\left(u_{\varepsilon}-\bar{u}_{\varepsilon}\right) \mathrm{d} \sigma \mathrm{d} t+O\left(\varepsilon^{2 /(N+2)}\right)
\end{aligned}
$$

then, assuming that $u_{\delta}^{(1)} \in C^{1}(\bar{\Omega} \times[0, T])$ is such that $\left\|U_{0}-u_{\delta}^{(1)}\right\|_{L^{2}(\Omega \times[0, T])} \leq \delta$, we get, by using Lemma 10, Lemma 11 (convergence of $u_{\varepsilon}$ to $U_{0}$ in $L^{2}(\Omega \times[0, T])$ ), continuity properties of $g(u, y)$ and $g_{u}^{\prime}(u, y)$ in $u$ (conditions (2.3), (2.4), (2.5)), (4.3) and the second bound in (6.9),

$$
\begin{aligned}
\lim _{\varepsilon \rightarrow 0} \int_{0}^{T}\left\langle\mathcal{G}_{\varepsilon}\left(u_{\varepsilon}\right), u_{\varepsilon}-v_{\varepsilon}\right\rangle_{\varepsilon} \mathrm{d} t & =\lim _{\varepsilon \rightarrow 0} \int_{0}^{T} \int_{S_{\varepsilon}}\left(g\left(u_{\delta}^{(1)}, x / \varepsilon\right)\left(u_{\varepsilon}-v_{\varepsilon}-\bar{u}_{\varepsilon}+\bar{v}_{\varepsilon}\right)\right. \\
& \left.+g_{u}^{\prime}\left(u_{\delta}^{(1)}, x / \varepsilon\right)\left(u_{\delta}^{(1)}-V_{0}\right)\left(u_{\varepsilon}-\bar{u}_{\varepsilon}\right)\right) \mathrm{d} \sigma \mathrm{d} t+O(\delta),
\end{aligned}
$$

provided that the limits exist. By using Proposition 12 we identify the limits in the r.h.s. of (6.27) and then obtain (6.22) by passing to the limit $\delta \rightarrow 0$. 
Now, thanks to the monotonicity of the operator $\mathcal{A}_{\varepsilon}(u)$ we can take $\liminf \operatorname{in}_{\varepsilon \rightarrow 0}$ in (6.19) to obtain by virtue of (6.20), (6.21), (6.22) that

$$
\begin{aligned}
\int_{0}^{T}\left(\left\langle\partial_{t} U_{0}(t), U_{0}(t)-V_{0}(t)\right\rangle-\left\langle f(t), U_{0}(t)-V_{0}(t)\right\rangle\right) \mathrm{d} t \\
+\int_{0}^{T} \int_{\Omega} \int_{Y^{*}} a\left(D_{x} V_{0}+D_{y} V_{1}, y\right) \cdot\left(D_{x} U_{0}+D_{y} U_{1}-D_{x} V_{0}-D_{y} V_{1}\right) \mathrm{d} y \mathrm{~d} x \mathrm{~d} t \\
-\int_{0}^{T} M\left(U_{0}, U_{1}, V_{0}, V_{1}\right) \mathrm{d} t \leq 0 .
\end{aligned}
$$

This inequality is shown for any $V_{0}(x, t) \in C^{\infty}(\bar{\Omega} \times[0, T])$ and any $V_{1}(x, y, t) \in$ $C^{\infty}(\bar{\Omega} \times \bar{Y} \times[0, T])(Y$-periodic in $y)$, by an approximation argument it still holds for any $V_{0} \in L^{2}\left(0, T ; W^{1,2}(\Omega)\right), V_{1} \in L^{2}\left(0, T ; L^{2}\left(\Omega ; W_{\text {per }}^{1,2}(Y)\right)\right)$. Therefore we can set $V_{0}=U_{0}, V_{1}=U_{1} \pm \delta \phi(x, t) w(y)$, where $w \in W_{\text {per }}^{1,2}(Y), \phi \in C^{\infty}(\bar{\Omega} \times[0, T])$ and $\delta>0$ are arbitrary, divide (6.28) by $\delta$ and pass to the limit as $\delta \rightarrow 0$ to get,

$$
\int_{0}^{T} \int_{\Omega}\left(\int_{Y^{*}} a\left(D_{x} U_{0}+D_{y} U_{1}, y\right) \cdot D_{y} w \mathrm{~d} y-\int_{S \cap Y} g\left(U_{0}, y\right) w \mathrm{~d} \sigma_{y}\right) \varphi(x, t) \mathrm{d} x \mathrm{~d} t=0 .
$$

This means, that $U_{1}$ solves (2.13) with $u=U_{0}$ and $\xi=D_{x} U_{0}$ for almost all $(x, t) \in$ $\Omega \times[0, T]$. Now set $V_{0}=U_{0} \pm \delta \Phi(x, t), V_{1}=U_{1}$, where $\Phi \in C^{\infty}(\bar{\Omega} \times[0, T])$ and $\delta>0$ are arbitrary, divide (6.28) by $\delta$ and pass to the limit as $\delta \rightarrow 0$. As a result we obtain

$$
\begin{aligned}
&\left|Y^{*}\right| \int_{0}^{T} \int_{\Omega} \partial_{t} U_{0}(x, \tau) \Phi(x, \tau) \mathrm{d} x \mathrm{~d} \tau \\
&+\int_{0}^{T} \int_{\Omega}\left(a^{*}\left(D_{x} U_{0}, U_{0}\right) \cdot D_{x} \Phi-b^{*}\left(D_{x} U_{0}, U_{0}\right) \Phi-\operatorname{div}_{x}\left(g^{*}\left(U_{0}\right) \Phi\right)\right) \mathrm{d} x \mathrm{~d} \tau \\
&=\left|Y^{*}\right| \int_{0}^{T} \int_{\Omega} f(x, t) \Phi(x, \tau) \mathrm{d} x \mathrm{~d} \tau
\end{aligned}
$$

this yields (1.4).

\section{Properties of the homogenized problem}

Define the operators $\mathcal{A}^{*}, \mathcal{B}^{*}, \mathcal{T}^{*}: X \rightarrow X^{*}$ by $\mathcal{B}^{*}(u)=b^{*}(D u, u)$,

$$
\left\langle\mathcal{A}^{*}(u), v\right\rangle=\int_{\Omega} a^{*}(D u, u) \cdot D v \mathrm{~d} x, \forall v \in X,
$$




$$
\left\langle\mathcal{T}^{*}(u), v\right\rangle=\int_{\partial \Omega} g^{*}(u) \cdot \nu v \mathrm{~d} \sigma=\int_{\Omega} \operatorname{div}\left(g^{*}(u) v\right) \mathrm{d} x, \forall v \in X .
$$

Then, in terms of the operator $\mathcal{F}^{*}(u)=\mathcal{A}^{*}(u)-\mathcal{B}^{*}(u)-\mathcal{T}^{*}(u)$, problems (1.3) and (1.4) read

$$
\begin{gathered}
\mathcal{F}^{*}(u)+\lambda u=f, \\
\left\{\begin{array}{l}
\partial_{t} u+\mathcal{F}^{*}(u)=f, t>0 \\
u=\tilde{u}, \text { when } t=0 .
\end{array}\right.
\end{gathered}
$$

According to Theorem 2 there is a solution (obtained as the limit of solutions of (1.1)) of (7.1) for every $f \in L^{2}(\Omega)$; similarly, by Theorem 6 problem (17.2) has a solution on the time interval $[0, T]$ when $f \in L^{2}(\Omega \times[0, T])$ and $\tilde{u} \in L^{2}(\Omega)$. The solvability of problems (7.1) and (7.2) can be proved for more general $f$, namely, we can assume merely $f \in X^{*}$ and $f \in L^{2}\left(0, T ; X^{*}\right)$ in (17.1) and (7.2), respectively. However we will focus on the uniqueness results.

$\mathbf{1}\left(\right.$ Properties of $a^{*}$ and $\left.b^{*}\right)$. First we show

Lemma 13. The functions $a^{*}$ and $b^{*}$ given by (2.10), (2.11) are continuous. Moreover, there are constants $\gamma, \alpha, r>0$ and $C$ such that

$$
\begin{gathered}
a^{*}(\xi, u) \cdot \xi \geq \gamma|\xi|^{2}-C\left(|u|^{2}+1\right) \text { and }\left|a^{*}(\xi, u)\right| \leq C(|\xi|+|u|+1), \\
\left(a^{*}(\xi, u)-a^{*}(\zeta, v)\right) \cdot(\xi-\zeta) \geq \alpha|\xi-\zeta|^{2}-r(u-v)^{2}, \\
\left|b^{*}(\xi, u)\right| \leq C(|\xi|+|u|+1) \text { and } \\
\left.\left(b^{*}(\xi, u)-b^{*}(\zeta, v)\right)(v-u) \leq \frac{1}{4}\left(a^{*}(\xi, u)-a^{*}(\zeta, v)\right) \cdot(\xi-\zeta)\right) \\
+C\left(|u-v|^{2}+|u-v|^{2}(|\xi|+|u|+1) /(1+|u-v|)\right) .
\end{gathered}
$$

The proof of this Lemma is based on the study of properties of solutions $w(y ; \xi, u)$ of problem (2.13). We will make use of the following well-known results,

$$
\begin{gathered}
\int_{S \cap Y}\left|w-\frac{1}{\left|Y^{*}\right|} \int_{Y^{*}} w \mathrm{~d} x\right|^{2} \mathrm{~d} \sigma \leq C \int_{Y^{*}}|D w|^{2} \mathrm{~d} x, \\
\int_{Y^{*}}\left|D_{y} w+\xi\right|^{2} \mathrm{~d} y \geq \rho|\xi|^{2}, \rho>0
\end{gathered}
$$

for all $\xi \in \mathbb{R}^{N}, w \in W_{\text {per }}^{1,2}\left(Y^{*}\right)$, where $C$ and $\rho$ are independent of $w$ and $\xi$. 
Lemma 14. For any $\xi \in \mathbb{R}^{N}, u \in \mathbb{R}$ there is a unique (modulo an additive constant) solution $w(y ; \xi, u)$ of problem (2.13) and we have

(a) $\int_{Y^{*}}\left|D_{y} w(y ; \xi, u)\right|^{2} \mathrm{~d} y \leq C\left(|\xi|^{2}+|u|^{2}+1\right)$,

(b) $a^{*}(\xi, u) \cdot \xi \geq \gamma|\xi|^{2}-C\left(|u||\xi|+|u|^{2}+1\right)($ with $\gamma>0)$,

(c) there are $\alpha, \beta>0$ and $r$ such that, for any $\xi, \zeta \in \mathbb{R}^{N}$ and $u, v \in \mathbb{R}$

$$
\left(a^{*}(\xi, u)-a^{*}(\zeta, v)\right) \cdot(\xi-\zeta) \geq \alpha|\xi-\zeta|^{2}-r(u-v)^{2}+\beta \int_{Y^{*}}|D \hat{w}|^{2} \mathrm{~d} y,
$$

where $\hat{w}=w(y ; \xi, u)-w(y ; \zeta, v)$,

(d) $w(y ; \zeta, v) \rightarrow w(y ; \xi, u)$ strongly in $W_{\text {per }}^{1,2}\left(Y^{*}\right) \backslash \mathbb{R}$ when $\zeta \rightarrow \xi, v \rightarrow u$.

Proof. The existence of a unique solution of $(2.13)$ in $W_{\text {per }}^{1,2}\left(Y^{*}\right) \backslash \mathbb{R}$ easily follows from assumptions (i)-(iii) and (vi) on the functions $a$ and $g$. To show (a) we derive from (2.13) by integrating by parts

$$
\int_{Y^{*}} a(\xi+D w, y) \cdot(\xi+D w) \mathrm{d} y=\int_{S \cap Y} g(u, y) w \mathrm{~d} \sigma+\int_{Y^{*}} a(\xi+D w, y) \cdot \xi \mathrm{d} y
$$

By applying the Poincaré inequality (7.6) and taking into account (2.6), (2.3) we obtain that for any $k>0$,

$$
\begin{aligned}
& \int_{Y^{*}} a(\xi+D w, y) \cdot(\xi+D w) \mathrm{d} y \leq C(|u|+1)\|D w\|_{L^{2}\left(Y^{*}\right)}+C|\xi|\|\xi+D w\|_{L^{2}\left(Y^{*}\right)} \\
& \leq C(|u|+1)\left(\|\xi+D w\|_{L^{2}\left(Y^{*}\right)}+|\xi|\right)+C|\xi|\|\xi+D w\|_{L^{2}\left(Y^{*}\right)} \\
& \leq k\left((|u|+1)^{2}+\frac{C}{k}\left(|\xi|^{2}+\|\xi+D w\|_{L^{2}\left(Y^{*}\right)}^{2}\right),\right.
\end{aligned}
$$

where $C$ is independent of $k, u$ and $\xi$. If we choose $k$ in (7.9) large enough and use (2.2) we get

$$
\int_{Y^{*}}|\xi+D w|^{2} \mathrm{~d} y \leq C\left(|u|^{2}+|\xi|^{2}+1\right)
$$

that in turn implies (a).

By using (17.7) on the l.h.s. of (7.8) and (7.6) in conjunction with (2.3), (2.6) in the first term of the r.h.s., we easily derive (b). 
In order to show (c) we use (2.13) to get by integrating by parts

$$
\begin{aligned}
& \left(a^{*}(\xi, u)-a^{*}(\zeta, v)\right) \cdot(\xi-\zeta)=\int_{S \cap Y}(g(v, y)-g(u, y)) \hat{w} \mathrm{~d} \sigma \\
& \quad+\int_{Y^{*}}\left(a\left(\xi+D_{y} w(y ; \xi, u)\right)-a\left(\zeta+D_{y} w(y ; \zeta, v)\right)\right) \cdot\left(\xi-\zeta+D_{y} \hat{w}\right) \mathrm{d} y
\end{aligned}
$$

Taking into account (2.4), (2.6) and applying (7.6) we can estimate the first term $I_{1}$ on the r.h.s. of $(7.10)$ as

$$
\left|I_{1}\right| \leq k|u-v|^{2}+\frac{C}{k} \int_{Y^{*}}|D \hat{w}|^{2} \mathrm{~d} y, \text { for any } r>0,
$$

where $C$ is independent of $k, \xi, \zeta, u, v$. In view of (2.1) and (7.7) we have the following lower bound for the second term $I_{2}$ in (7.10)

$$
I_{2} \geq(1-\delta) \kappa \rho|\xi-\zeta|^{2}+\delta \kappa \int_{Y^{*}}\left|\xi-\zeta+D_{y} \hat{w}\right|^{2} \mathrm{~d} y
$$

with $0<\delta<1$ to be chosen later. On the other hand, by the elementary inequality $a^{2} \leq 2(a+b)^{2}+2 b^{2}$,

$$
\int_{Y^{*}}\left|D_{y} \hat{w}\right|^{2} \mathrm{~d} y \leq 2 \int_{Y^{*}}\left|\xi-\zeta+D_{y} \hat{w}\right|^{2} \mathrm{~d} y+2|\xi-\zeta|^{2},
$$

thus

$$
I_{2} \geq \kappa(\rho-\delta(\rho+1))|\xi-\zeta|^{2}+\frac{\delta \kappa}{2} \int_{Y^{*}}\left|D_{y} \hat{w}\right|^{2} \mathrm{~d} y
$$

Choose $0<\delta<1$ so that $\rho-\delta(æ+1)>0$ and set $k=4 C /(\delta \kappa)$ (where $C$ is the constant appearing in (7.11) $)$, we thus obtain (b) with $\alpha=\kappa(\rho-\delta(\rho+1))>0$, $\beta=(\delta \kappa) / 4>0$.

Finally, statement $(d)$ is a direct consequence of (a) and (c).

Proof of Lemma 13. According to Lemma 14 it suffices only to show (7.5). Set $\hat{w}=w(y ; \xi, u)-w(y ; \zeta, v)$, we have by using (7.6) and assumptions (i), (iii), (iv) on $g$,

$$
\begin{aligned}
& \left(b^{*}(\xi, u)-b^{*}(\zeta, v)\right)(v-u)=(v-u) \int_{S \cap Y} g_{u}^{\prime}(v, y) \hat{w} \mathrm{~d} \sigma_{y} \\
& \quad+(v-u) \int_{S \cap Y}\left(g_{u}^{\prime}(u, y)-g_{u}^{\prime}(v, y)\right) w(y ; \xi, u) \mathrm{d} \sigma_{y} \\
& \quad \leq C|u-v|\|D \hat{w}\|_{L^{2}\left(Y^{*}\right)}+C|u-v|^{2}\|D w(\cdot ; \xi, u)\|_{L^{2}\left(Y^{*}\right)} /(1+|u|+|v|) .
\end{aligned}
$$

Then statements (a) and (c) of Lemma 14 yield (7.5). 
Remark 15. In the case when the function $g(u, y)$ is linear in $u$, bound (7.5) simplifies to the following one,

$$
\left.\left(b^{*}(\xi, u)-b^{*}(\zeta, v)\right)(v-u) \leq \frac{1}{4}\left(a^{*}(\xi, u)-a^{*}(\zeta, v)\right) \cdot(\xi-\zeta)\right)+C|u-v|^{2} .
$$

Let us consider next the particular case when $a(\xi, y)$ is linear in $\xi$, i.e. $a$ is given by $a(\xi, y)=A(y) \xi$ with $A \in L^{\infty}\left(Y ; \mathbb{R}^{N \times N}\right), A(y) \xi \cdot \xi \geq \kappa|\xi|^{2}(\kappa>0)$, $\forall \xi \in \mathbb{R}^{N}, y \in Y$. Then we can write the solution of (2.13) as the sum $w(y ; \xi, u)=$ $w^{(1)}(y ; \xi)+\tilde{w}(y ; u)$ with $w^{(1)}$ solving (2.13) and $\tilde{w}$ being a unique (up to an additive constant) solution of

$$
\left\{\begin{array}{l}
\operatorname{div}\left(A(y) D_{y} \tilde{w}\right)=0 \text { in } Y^{*} \\
A(y) D_{y} \tilde{w} \cdot \nu=g(u, y) \text { on } S \cap Y \\
\tilde{w} \text { is } Y \text {-periodic. }
\end{array}\right.
$$

Note that $w^{(1)}(y ; \xi)$ depends linearly on $\xi$, also we have

$$
\begin{aligned}
& \|\tilde{w}(y ; u)\|_{W^{1,2}\left(Y^{*}\right) \backslash \mathbb{R}} \leq C(|u|+1), \| \tilde{w}(y ; u)-\tilde{w}\left(y ; v \|_{W^{1,2}\left(Y^{*}\right) \backslash \mathbb{R}} \leq C|u-v|,\right. \\
& \left\|\tilde{w}_{u}^{\prime}(y ; u)-\tilde{w}_{u}^{\prime}(y ; v)\right\|_{W^{1,2)}\left(Y^{*}\right) \backslash \mathbb{R}} \leq C|u-v| /(1+|u|+|v|),
\end{aligned}
$$

where $C$ is independent of $u, v$. The proof of these bounds is analogous to that of (3.14) - (3.16). Thus we have

$$
\begin{array}{rl}
b^{*}(\xi, u)=\frac{\partial}{\partial u} \int_{Y^{*}} & A(y) D_{y} \tilde{w}(y ; u) \cdot D_{y} w^{(1)}(y ; \xi) \mathrm{d} y \\
& \quad+\int_{Y^{*}} A(y) D_{y} \tilde{w}_{u}^{\prime}(y ; u) \cdot D_{y} \tilde{w}(y ; u) \mathrm{d} y=H^{\prime}(u) \cdot \xi+h(u)
\end{array}
$$

with $H, h$ such that $|H(u)-H(v)| \leq C|u-v|,|h(u)-h(v)| \leq C|u-v|$.

2 (Uniqueness results for problem (17.1)). In the particular cases when the dimension of the space $N \leq 3$ or $a(\xi, y)$ is linear in $\xi$ or $g(u, y)$ is linear in $u$ we show that problem (7.1) cannot have two distinct solutions for sufficiently large $\lambda$.

The following inequality will be used to estimate the expressions involving traces on $\partial \Omega$. For every $\delta>0$ there is $\Lambda_{\delta}$ such that

$$
\int_{\partial \Omega}|w|^{2} \mathrm{~d} \sigma \leq \delta\|D w\|_{L^{2}(\Omega)}^{2}+\Lambda_{\delta}\|w\|_{L^{2}(\Omega)}, \forall w \in W^{1,2}(\Omega) .
$$


This inequality is a consequence of the compactness of the trace operator $T_{\partial \Omega}$ : $W^{1,2}(\Omega) \rightarrow L^{2}(\partial \Omega), T_{\partial \Omega} u=$ trace of $u$ on $\partial \Omega$. Thanks to the Lipschitz continuity of $g(u, y)$ in the variable $u$, inequality (7.15) implies that

$$
\left|\left\langle\mathcal{T}^{*}(u)-\mathcal{T}^{*}(v), u-v\right\rangle\right| \leq \frac{\alpha}{4}\|u-v\|_{X}^{2}+C\|u-v\|_{L^{2}(\Omega)}^{2},
$$

where $\alpha>0$ is the same as in (7.4).

Let $u, v$ be solutions of (7.1).

Case I $(g(u, y)$ is linear in $u)$. By using Lemma 13, Remark 15] and (7.16) we get

$$
\left\langle\mathcal{F}^{*}(u)-\mathcal{F}^{*}(v)+\lambda(u-v), u-v\right\rangle \geq \frac{\alpha}{4}\|u-v\|_{X}^{2}+\left(\lambda-\hat{\lambda}_{0}\right)\|u-v\|_{L^{2}(\Omega)}^{2},
$$

with $\hat{\lambda}_{0}$ independent of $\lambda$. It follows that $u=v$ if $\lambda \geq \hat{\lambda}_{0}$.

Case II $(a(\xi, y)$ is linear in $\xi)$. We have, according to (17.14),

$$
\begin{gathered}
\left.\left\langle B^{*}(u)-\mathcal{B}^{*}(v)\right), v-u\right\rangle=\left|Y^{*}\right| \int_{\Omega}(u-v)(\operatorname{div}(H(u)-H(v))+h(u)-h(v)) \mathrm{d} x \\
=\left|Y^{*}\right| \int_{\Omega}(D(v-u) \cdot(H(u)-H(v))+(u-v)(h(u)-h(v))) \mathrm{d} x \\
+\left|Y^{*}\right| \int_{\partial \Omega}(u-v)(H(u)-H(v)) \cdot \nu \mathrm{d} \sigma \\
\leq \frac{\alpha}{4}\|u-v\|_{X}^{2}+C\|u-v\|_{L^{2}(\Omega)}^{2},
\end{gathered}
$$

where we have used (7.15). This inequality and Lemma13 yield (7.17) (with possibly another constant $\hat{\lambda}_{0}$ ).

Case III (The space dimension $N$ is two or three). It is well known that for these space dimensions $X\left(=W^{1,2}(\Omega)\right)$ is compactly embedded into $L^{4}(\Omega)$, moreover $\|w\|_{L^{4}(\Omega)}^{2} \leq C \delta\|w\|_{X}^{2}+C \delta^{-N /(4-N)}\|w\|_{L^{2}(\Omega)}^{2}$ for all $w \in X$ and $\delta>0$, where $C$ is independent of $\delta>0$ and $w$ (see, e.g., [12]). By using this inequality, Lemma 13 and (7.16) we easily show that

$$
\begin{aligned}
& \left\langle\mathcal{F}^{*}(u)-\mathcal{F}^{*}(v), u-v\right\rangle \geq \frac{\alpha}{4}\|u-v\|_{X}^{2} \\
& \quad-C\left(\delta\|u-v\|_{X}^{2}+\delta^{-N /(4-N)}\|u-v\|_{L^{2}(\Omega)}^{2}\right)\left(\|u\|_{X}+1\right), \quad \forall \delta>0 .
\end{aligned}
$$

On the other hand Lemma 13 and the very definition of $\mathcal{T}^{*}(u)$ imply that for every $w \in X\left\langle\mathcal{A}^{*}(w), w\right\rangle \geq \gamma\|w\|_{X}^{2}-C\left(\|w\|_{L^{2}(\Omega)}^{2}+1\right),\left|\left\langle\mathcal{B}^{*}(w), w\right\rangle\right| \leq C\left(\|w\|_{X}+\|w\|_{L^{2}(\Omega)}+\right.$ 
1) $\|w\|_{L^{2}(\Omega)}$ and $\left|\left\langle\mathcal{T}^{*}(w), w\right\rangle\right| \leq C\|w\|_{X}\|w\|_{L^{2}(\Omega)}$. Therefore there is $\tilde{\lambda}_{0}$ such that $\left\langle F^{*}(u), u\right\rangle \geq \frac{\gamma}{2}\|u\|_{X}^{2}-\tilde{\lambda}_{0}\langle u, u\rangle$, hence, for $\lambda \geq \tilde{\lambda}_{0}$ we have the a-priori bound $\|u\|_{X} \leq$ $C\left(\|f\|_{X^{*}}+1\right)$ with $C$ independent of $u, f$ and $\lambda \geq \tilde{\lambda}_{0}$. Thus, $u$ and $v$ being solutions of (7.1), estimate (17.18) yields

$$
\frac{\alpha}{4}\|u-v\|_{X}^{2}+\lambda\|u-v\|_{L^{2}(\Omega)}^{2} \leq C\left(\|f\|_{X^{*}}+1\right)\left(\delta\|u-v\|_{X}^{2}+\delta^{-N /(4-N)}\|u-v\|_{L^{2}(\Omega)}^{2}\right),
$$

and by setting $\delta=\alpha /\left(8 C\left(\left(\|f\|_{X^{*}}+2\right)\right)\right.$ we get $u=v$ as far as $\lambda \geq \hat{\lambda}_{0}(=$ $\left.\max \left\{\tilde{\lambda}_{0}, C\left(\|f\|_{X^{*}}+1\right) \delta^{-N /(4-N)}\right\}\right)$. ( $\hat{\lambda}_{0}$ can be chosen independent of $f$ if $N=2$.)

2(Uniqueness results for problem (7.2)). Given $T>0$, we show that problem (7.2) cannot have two distinct solutions $u, v$ on the time interval $[0, T]$ if $a(\xi, y)$ is linear in $\xi$ or $g(u, y)$ is linear in $u$. Indeed, $w=u-v$ satisfies $\partial_{t}\langle w(t), w(t)\rangle+2\left\langle\mathcal{F}^{*}(u(t))-\right.$ $\left.\mathcal{F}^{*}(v(t)), u(t)-v(t)\right\rangle=0,0<t<T$, and $w(0)=0$, while (7.17) yields $-2\left\langle\mathcal{F}^{*}(u(t))-\right.$ $\left.\mathcal{F}^{*}(v(t)), u(t)-v(t)\right\rangle \leq C\langle w(t), w(t)\rangle, 0<t<T$, therefore $e^{-C t}\|w(t)\|_{L^{2}(\Omega)}^{2} \leq 0$ so that $w \equiv 0$.

In the case when space dimension is two we also have the uniqueness result. Note that we have at least one solution $u \in L^{2}(0, T ; X)$ of (7.2). Then, if $v$ is another solution we set $w=u-v, R(t)=\langle w(t), w(t)\rangle$, and derive by using (7.18) with $\delta=\alpha /\left(8 C\left(\left(\|u\|_{X}+1\right)\right)\right.$,

$$
R^{\prime}(t)-C R(t)\left(\|u(t)\|_{X}+1\right)^{2} \leq 0,0<t<T, \text { and } R(0)=0 .
$$

This implies that $R(t) \exp \left\{-C \int_{0}^{t}\left(\|u(\tau)\|_{X}+1\right)^{2} \mathrm{~d} \tau\right\} \leq 0$ and therefore $R \equiv 0$, i.e. $u=v$.

Acknowledgments. The work of V.Rybalko is partially supported by the Grant of NASU for Young Scientists. This work was originated and partially done when V.Rybalko enjoyed the hospitality of the Narvik University College whose support is gratefully acknowledged.

\section{References}

[1] G. Allaire, Homogenization and two-scale convergence. SIAM J. Math. Anal. 23 (1992), no. 6, 1482-1518. 
[2] E. Acerbi, V. Chiado Piat;G. Dal Maso,D. Percivale, An extension theorem from connected sets, and homogenization in general periodic domains. Nonlinear Anal. 18 (1992), no. 5, 481-496.

[3] G. Allaire,A. Damlamian,U. Hornung, Two-scale convergence on periodic surfaces and applications, in: Proceedings of the International Conference on Mathematical Modelling of Flow Through Porous Media, World Scientific Publications, Singapore, 1995, pp. 15-25.

[4] A.G. Belyaev, A.L. Pyatnitskii, G.A. Chechkin, Asymptotic behavior of the solution of a boundary value problem in a punctured domain with an oscillating boundary. (Russian) Sibirsk. Mat. Zh. 39 (1998), no. 4, 730-754; translation in Siberian Math. J. 39 (1998), no. 4, 621-644

[5] A.G. Belyaev, A.L. Pyatnitskii, G.A. Chechkin, Averaging in a perforated domain with an oscillating third boundary condition. (Russian) Mat. Sb. 192 (2001), no. 7, 3-20; translation in Sb. Math. 192 (2001), no. 7-8, 933-949

[6] V. Chiado Piat, A. Piatniski, Gamma-convergence approach to variational problems in perforated domains with Fourier boundary conditions, COCV (2008), DOI:10.1051/COCV:2008073.

[7] D.Cioranescu, P.Donato, On a Robin problem in perforated domains D. Cioranescu, P. Donato, On a Robin problem in perforated domains. Homogenization and applications to material sciences (Nice, 1995), 123-135, GAKUTO Internat. Ser. Math. Sci. Appl., 9, Gakkotosho, Tokyo, 1995

[8] D. Cioranescu, J. Saint Jean Paulin, Truss structures: Fourier conditions and eigenvalue problem. Boundary control and boundary variation (Sophia-Antipolis, 1990), 125-141, Lecture Notes in Control and Inform. Sci., 178, Springer, Berlin, 1992

[9] G. Clark, L. Packer. Two-scale homogenization of non-linear degenerate evolution equations. J. Math. Anal. Appl. 238 (1999), no. 1, 316-328.

[10] G. Clark, L. Packer. Two-scale homogenization of implicit degenerate evolution equations. J. Math. Anal. Appl. 214 (1997), no. 2, 420-438.

[11] G. Clark, R. Showalter. Two-scale convergence of a model for flow in a partially fissured medium. Electron. J. Differential Equations 1999, No. 2, 20 pp. (electronic). 
[12] O.A. Ladyzhenskaya, N.N. Uraltseva. Linear and quasilinear elliptic equations. Translated from the Russian by Scripta Technica, Inc. Translation editor: Leon Ehrenpreis Academic Press, New York-London 1968 xviii+495 pp. 35.47

[13] D. Lukkassen, G. Nguestseng, P. Wall, Two-scale convergence. Int. J. Pure Appl. Math. 2 (2002), no. 1, 35-86.

[14] G. Nguetseng, A general convergence result for a functional related to the theory of homogenization. SIAM J. Math. Anal. 20 (1989), no. 3, 608-623.

[15] O.A. Oleinik, T.A. Shaposhnikova, On the homogenization of the Poisson equation in partially perforated domains with arbitrary density of cavities and mixed type conditions on their boundary. Atti Accad. Naz. Lincei Cl. Sci. Fis. Mat. Natur. Rend. Lincei (9) Mat. Appl. 7 (1996), no. 3, 129-146.

[16] O.A. Oleinik, T.A. Shaposhnikova, On an averaging problem in a partially punctured domain with a boundary condition of mixed type on the boundary of the holes, containing a small parameter. (Russian) Differ. Uravn. 31 (1995), no. 7, 1150-1160, 1268; translation in Differential Equations 31 (1995), no. 7, 1086-1098 (1996)

[17] L. Pankratov, A. Piatniskii, V. Rybalko. Homogenized model of reaction-diffusion in a porous medium, C.R. Mecanique 331 (2003), 253-258.

[18] S.E. Pastukhova, On the character of the distribution of the temperature field in a perforated body with a given value on the outer boundary under heat exchange conditions on the boundary of the cavities that are in accord with Newton's law. (Russian) Mat. Sb. 187 (1996), no. 6, 85-96; translation in Sb. Math. 187 (1996), no. $6,753-770$

[19] S.E. Pastukhova, Tartar's compensated compactness method in the averaging of the spectrum of a mixed problem for an elliptic equation in a punctured domain with a third boundary condition. (Russian. Russian summary) Mat. Sb. 186 (1995), no. 5, 127-144; translation in Sb. Math. 186 (1995), no. 5, 753-770

[20] S.E. Pastukhova, Spectral asymptotics for a stationary heat conduction problem in a perforated domain. (Russian. Russian summary) Mat. Zametki 69 (2001), no. 4, 600-612; translation in Math. Notes 69 (2001), no. 3-4, 546-558 
[21] R.E. Showalter. Monotone operators in Banach space and nonlinear partial differential equations. Mathematical Surveys and Monographs, 49. American Mathematical Society, Providence, RI, 1997. xiv+278 pp. ISBN: 0-8218-0500-2 